\title{
Putting Ourselves in Another's Skin: Using the Plasticity of Self-Perception to Enhance Empathy and Decrease Prejudice
}

\author{
Harry Farmer ${ }^{1,2}$ (D) Lara Maister $^{3,4}$
}

Published online: 31 October 2017

(C) The Author(s) 2017. This article is an open access publication

\begin{abstract}
The self is one the most important concepts in social cognition and plays a crucial role in determining questions such as which social groups we view ourselves as belonging to and how we relate to others. In the past decade, the self has also become an important topic within cognitive neuroscience with an explosion in the number of studies seeking to understand how different aspects of the self are represented within the brain. In this paper, we first outline the recent research on the neurocognitive basis of the self and highlight a key distinction between two forms of self-representation. The first is the "bodily" self, which is thought to be the basis of subjective experience and is grounded in the processing of sensorimotor signals. The second is the "conceptual" self, which develops through our interactions of other and is formed of a rich network of associative and semantic information. We then investigate how both the bodily and conceptual self are related to social cognition with an emphasis on how self-representations are involved in the processing and creation of prejudice. We then highlight new research demonstrating that the bodily and conceptual self are both malleable and that this malleability can be harnessed in order to achieve a reduction in social prejudice. In particular, we will outline strong evidence that modulating people's perceptions of the bodily self
\end{abstract}

\section{Harry Farmer}

h.farmer@ucl.ac.uk

Lara Maister

lara.maister@bbk.ac.uk

1 Institute of Cognitive Neuroscience, University College London, 17 Queen Square, London WC1N 3AR, UK

2 Department of Psychology, University of Bath, Claverton Down, Bath BA2 7AY, UK

3 Department of Psychology, Royal Holloway, University of London, Egham, Surrey TW20 0EX, UK

4 Department of Psychological Sciences, Birbeck, University of London, Malet Street, London WC1E 7HX, UK 
can lead to changes in attitudes at the conceptual level. We will highlight a series of studies demonstrating that social attitudes towards various social out-groups (e.g. racial groups) can lead to a reduction in prejudice towards that group. Finally, we seek to place these findings in a broader social context by considering how innovations in virtual reality technology can allow experiences of taking on another's identity are likely to become both more commonplace and more convincing in the future and the various opportunities and risks associated with using such technology to reduce prejudice.

Keywords Self · Body representation · Prejudice · Virtual reality · Social cognition

\section{Introduction}

The self is one the most important concepts in social cognition and plays a crucial role in determining which social groups we view ourselves as belonging to and how we relate to others. In the past decade, the self has also become an important topic within cognitive neuroscience, with an explosion in the number of studies seeking to understand how different aspects of the self are represented within the brain. In this paper, we first outline the recent research on the neurocognitive basis of the self and highlight a key distinction between bodily and conceptual forms of self-representation. We then move on to discuss the social nature of the self and how both bodily and conceptual forms of selfhood are closely tied to how we relate to others, with an emphasis on how such relations are affected by social prejudice. Finally, we highlight exciting recent research demonstrating the plasticity of self-representation and examine how altering self-perception may provide novel techniques that can increase empathy and decrease prejudice towards other social groups.

\section{The Self: Bodily and Conceptual}

The concept of the self has a long history in philosophy and the sciences, and our attempts to characterise it have led theorists to distinguish between many different forms of self. However, one clear distinction that emerges when reviewing the literature on the self is that between the "bodily" self and the "conceptual" self. The bodily self is thought to be the basis of subjective experience and is grounded in the brain's sensorimotor processing of the internal and external states of the body. In contrast, the conceptual self is formed of a rich network of associative and semantic information and develops through our interactions with others.

The distinction between these two important conceptions of selfhood can be found in thinkers across academic disciplines and traditions. Zahavi (2005) addresses this issue from the perspective of the phenomenological tradition and draws on the analyses made by Husserl (1982), Merleau-Ponty (1962) and Sartre (1956) to argue for the existence of both a phenomenal form of selfhood, which is 
considered to be an essential component of all subjective experience and to be strongly linked to our experience of our bodies, and a conceptual form of selfhood, which is greatly influenced by our interactions with others. A similar division of the self has also been put forward in analytic philosophy by Bermúdez (1998) and Strawson (1999) who both distinguish between forms of selfhood which require conceptual and linguistic mastery of the first person pronoun and a more basic nonconceptual self-consciousness, again assumed to rely primarily on sensorimotor signal from the body.

In the psychological sciences, James (2014) distinguished between the two senses of self, the "me self" which is the self experienced as object and can further be divided into material, social and spiritual aspects, and the "I self" or pure Ego, which is more fundamental and comprises the essence of lived subjective experience. In a similar manner, Neisser (1991) distinguishes between an ecological self which is developmentally primary and more advanced forms of selfhood such as possession of an extended self or a self-concept which require the ability to reflect on one's experiences over long time periods and to be able to identify one's self as part of a larger social group, respectively. Finally based on empirical findings, neuroscience researchers like Damasio (1999) and Edelman (2004) have distinguished between a continuously present form of self-awareness, based on the integration of diverse sensory and motor processes (which Damasio terms the "core self" and Edelman terms "primary consciousness") and a higher-level, temporally extended sense of self which plays a key role in social interaction and in our sense of personal identity. While the multifaceted nature of the concept of self means that any attempt at a binary classification will necessarily be crude and neglect some important nuances, for our current purposes we feel that the separation between bodily and conceptual selfhood captures an important conceptual distinction. In what follows, we will separately review current research regarding the theoretical and neural basis of these two forms of selfhood before moving on to discuss how both forms of the self can be linked to processes involved in social cognition.

\section{Bodily Self}

Of these two forms of self, the bodily self is the more fundamental in both developmental and experiential terms. The bodily self is considered to arise from the brain's integration of multiple signals from different sensory modalities into one coherent multimodal percept (De Vignemont, 2014; Hurley, 1998; Legrand, 2006).

This conception of the bodily self as the nexus of action and perception has also been put forward within the cognitive sciences. Neisser's (1991) account of the ecological self, which draws heavily on Gibson's (1979) theory of the importance of motor affordances in perception, views the ecological self as being specified largely by objective information which allows for the perception of both the location of the body and how it is interacting with the environment. Neisser considers this information to be largely dependent on knowledge of the body's movements and their relationship to vision. In addition to Neisser's focus on the interaction of action and perception, other researchers have emphasised the importance of internal bodily signals (or "interoception") in generating self-relevant bodily information (Craig, 
2003; Damasio, 1999; Seth, Suzuki, \& Critchley, 2011). These interoceptive senses include: proprioception or the movements of the musculoskeletal system (Fridland, 2011); the detection of the orientation of the body in space through the vestibular system (Pfeiffer et al., 2013); the neural and chemical signals indicating the state of one's cardiac, respiratory and digestive systems (Craig, 2003); and sensory signals for pain, itch, temperature and pleasant touch on the skin (McGlone \& Reilly, 2010). The sense of the bodily self is thought to arise when these sensory signals from within the body are then integrated with information from the external senses, primarily vision, to create a multisensory representation of the current state of the body (Seth et al., 2011).

For much of the history of cognitive science, there was no known methodology that allowed for the manipulation of people's experience of the bodily self. Although it was known that some neurological conditions could lead to somatoparaphrenia, the loss of a feeling of ownership over one's limbs (see Vallar \& Ronchi, 2009 for a review) examining this sense of body ownership in healthy participants seemed impossible due the difficulty in separating participants from what William James memorably described as "the feeling of the same old body always there" (James, 2014, p. 134). However, in the past decade, researchers have been able to carry out a large amount of research into body ownership thanks to a technique published by Botvinick and Cohen (1998). There, they showed that it is possible to induce a feeling of body ownership over an artificial hand by combining the visual stimulation of the prosthetic rubber hand with synchronous tactile stimulation of the participant's own hand. The resultant feeling of ownership over the rubber hand has led to this paradigm being known as the "rubber hand illusion" (RHI). This effect demonstrates that the experience of one's body as one's own depends upon the brain's ability to compare and integrate signals from different senses in a probabilistic manner (Apps \& Tsakiris, 2014; Limanowski \& Blankenburg, 2013). In the case of the RHI, the brain detects the synchrony between the felt touch on the real hand and the seen touch on the rubber hand and infers from this matching that the felt touch must originate from the observed hand leading to the feeling of ownership over that hand.

Since the discovery of the RHI, researchers have demonstrated that the integration of other body-related senses can also produce an illusory body ownership. These include: visual-motor synchrony, in which participants observe a hand moving in synch with one's own (Dummer, Picot-Annand, Neal, \& Moore, 2009; Riemer, Kleinböhl, Hölzl, \& Trojan, 2013; Walsh, Moseley, Taylor, \& Gandevia, 2011); tactile-motor synchrony in which touching a fake hand while one's own hand is touched leads to the perception that the participant is touching their own other hand (Lopez, Bieri, Preuss, \& Mast, 2012; White, Davies, \& Davies, 2011); the "rubber voice illusion" (Zheng, Macdonald, Munhall, \& Johnsrude, 2011) in which hearing a stranger speaking while saying the same words one's self led participants to experience the others voice as a distorted version of their own; between synchronous vision and pain stimulation (Capelari, Uribe, \& Brasil-Neto, 2009); and by synchronising the rhythm of visual flashes on the fake hand to the rhythm of participants' heartbeat. These last two are particularly important, as they involve the integration of an interoceptive signal (pain, or implicit awareness of the 
heartbeat) with an exteroceptive signal (vision). This lends support to models of self-awareness which make a link between representations of the internal state of the body and the bodily self (Craig, 2003; Damasio, 1999; Seth et al., 2011).

Further research has now extended the scope of body ownership, showing that synchronous visual-tactile stimulation can also generate illusions of ownership over other body parts in addition to the hand. For example, synchronous visual-tactile stimulation between one's own face and that of another can lead to an illusion of ownership over the other's face, as well as intriguing changes in the perceived similarity of that face to one's own (Sforza, Bufalari, Haggard, \& Aglioti, 2010; Tsakiris, 2008). Furthermore, body ownership illusions are no longer restricted to individual body parts; a "full-body illusion" has been developed, in which participants feel that they are embodied in a different spatial location to that of their own body (Ehrsson, 2007; Lenggenhager, Tadi, Metzinger, \& Blanke, 2007) and even that they have swapped bodies with another person (Petkova \& Ehrsson, 2008). These full-body illusions can also be generated by virtual reality, via visualmotor synchrony leading to embodiment of an avatar (Slater, Perez-Marcos, Ehrsson, \& Sanchez-Vives, 2009).

By combining these methodologies with neuroimaging techniques, it has been possible for cognitive neuroscientists to identify the brain areas that are integral for generating the sense of body ownership and which may be fundamental for the representation of the bodily self. These studies have revealed a network of uni- and multisensory brain regions that appear to play a role in the experience of body ownership. Some of these areas appear to reflect the specific properties of the body part being stimulated, for example, experiencing synchronous stimulation on the face leads to activation in face specific regions of the inferior occipital gyrus (Apps, Tajadura-Jiménez, Sereno, Blanke, \& Tsakiris, 2015), while stimulation of the hand or whole body leads to activation in a visual region that is sensitive to non-face body parts (Ionta et al., 2011; Limanowski, Lutti, \& Blankenburg, 2014). However, multisensory regions have also been identified which show sensitivity to the manipulation of body ownership, including the temporal-parietal junction (TPJ) (Ionta et al., 2011; Tsakiris, Costantini, \& Haggard, 2008), the dorsoanterior insula (Ehrsson, Spence, \& Passingham, 2004; Limanowski et al., 2014), the posterior insula (Tsakiris, Hesse, Boy, Haggard, \& Fink, 2007) and the premotor cortex (Bekrater-Bodmann et al., 2014; Ehrsson et al., 2004; Petkova et al., 2011; Tsakiris et al., 2007).

The findings of the involvement of both the TPJ and insula in the processing of the bodily self are of interest in terms of linking the bodily self to more socially and affectively relevant forms of self-representation as both have been associated with social and emotional cognitive processes. The TJP is activated during perspective taking and thinking about others (Carter \& Huettel, 2013; Santiesteban, Banissy, Catmur, \& Bird, 2012). The relationship between self-representation and the insula is more complex. Several recent meta-analyses of the insula (Chang, Yarkoni, Khaw, \& Sanfey, 2013; Deen, Pitskel, \& Pelphrey, 2011) have suggested evidence for a tripartite division between three areas: a posterior region (PI) which is thought to be a key hub for integrating booth internal and external signals relating to the body (Craig, 2009); a ventroanterior region (vAI) which is the region most strongly 
linked to the processing of emotional (Morita et al., 2013; Sanfey, Rilling, Aronson, Nystrom, \& Cohen, 2003) and social information (Lindner et al., 2013; Xiang, Lohrenz, \& Montague, 2013); and a dorsoanterior region (dAI) involved in higherlevel cognitive processing such as attention (Eckert et al., 2009). However, despite these functional differentiations, it is important to note that these areas show significant reciprocal connections between them with signals about the body processed in PI feeding into social-affective processing in vAI and into conscious awareness via attentional processing in the dAI (Craig, 2009; Simmons et al., 2013).

\section{Conceptual Self}

The conceptual self is generally thought to be made up of one's attitudes and beliefs about one's values, preferences, past experiences and social roles (Kuhl, Quirin, \& Koole, 2015). In contrast to the bodily self, the conceptual self is thought to emerge gradually during development via the association and organisation of intra- and interpersonal information closely related to one's behaviour and goals into a coherent network of self-relevant associations (Markus \& Wurf, 1987; Perugini \& Leone, 2009). The formation of the conceptual self relies on our interactions with others, particularly through linguistic and cultural identification (Gallagher, 2000; Gawronski \& Bodenhausen, 2006; Goffman, 1959; Mead, 1913, 1935; Neisser, 1991). In addition, the fact that the conceptual self is closely associated with autobiographical memories means that it plays a central role in the maintenance of personal identity over time (Charlesworth, Allen, \& Havelka, 2016; Damasio, 1999; Prebble, Addis, \& Tippett, 2013).

Social interaction appears to play a clear role in the development of the conceptual self. This aspect has been addressed by Nelson (2003), who has detailed how, as children begin to learn language, they are taught by caregivers to narrate the events of their lives. These narratives begin by being highly localised and simplistic, requiring a large amount of prompting from the care giver, e.g. "What sort of animals did you see at the circus?" (Fivush \& Nelson, 2006). However, over time children learn to develop these narratives on their own, which adds a new awareness of self in past and future experiences. This also allows them to develop the ability to contrast their own past and future self-narratives to those of others.

The type of scaffolding that the child receives plays a key role in shaping distinct variations in self-perception across culture and gender. For example, parents tend to be more elaborative when developing narratives with daughters than with sons which may explain why by their fourth year girls talk more about feelings and thoughts in personal experiences than boys (Fivush, 2011). Fivush and Nelson (2004) argue that the cultural differences in conceptions of selfhood between Western and Eastern cultures (for reviews see Cohen, Leung, \& Hoshino-Browne, 2007; Markus \& Kitayama, 2010) can be traced back to differences in the way that carers in the West and East support their children in developing narratives in autobiographical memory. They note findings that mothers from Western cultures talk about the past in more elaborate and emotional language and tend to focus on the child's own experience of events while mothers from Eastern cultures tend to emphasis the communal setting of events and to highlight the moral emotions and lessons that emerge from experiences 
(Leichtman, Wang, \& Pillmer, 2003). These differences in the style of scaffolding appear to continue across life with both older children and adults from Western cultures tending to have more detailed, elaborate and emotional narratives of their past than their counterparts in Eastern cultures (Pillmer, 1998).

Research in neuroscience on the conceptual self has largely focused on comparing neural activity when people are viewing stimuli related to self (e.g. self-relevant traits, own name, own face, autobiographical memories) as opposed to stimuli related to others. Several key regions along the cortical midline appear to show greater activation to self-related stimuli than other-related stimuli (Heatherton, 2006; Northoff et al., 2006; Northoff, Qin, \& Feinberg, 2011), including the anterior cingulate cortex (Leshikar, Cassidy, \& Gutchess, 2016), the medial prefrontal cortex (mPFC) and the vAI (Murray, Debbané, Fox, Bzdok, \& Eickhoff, 2015). The mPFC is particularly involved in the processing of information about people's traits, preferences and beliefs (Frith \& Frith, 2003; Ma, Baetens, Vandekerckhove, Van der Cruyssen, \& Van Overwalle, 2013; Schilbach, 2015) and shows sensitivity to a number of social factors including social status and ostracism (Muscatell et al., 2012; Powers, Wagner, Norris, \& Heatherton, 2013). Importantly, there is now considerable evidence for a ventral dorsal split within the mPFC, with the ventral region (vMPFC) showing greater involvement in the processing of self-relevant traits, while the dorsal region (dMPFC) is involving in processing the traits of dissimilar others (Araujo, Kaplan, \& Damasio, 2013; Denny, Kober, Wager, \& Ochsner, 2012; Mitchell, Macrae, \& Banaji, 2006; Moore, Merchant, Kahn, \& Pfeifer, 2014; Powell, Macrae, Cloutier, Metcalfe, \& Mitchell, 2010).

Further evidence for the role of the vmPFC in representing the conceptual self comes from studies showing cultural differences in vmPFC activation during selfprocessing. Zhu, Zhang, Fan, and Han (2007) scanned Western and Chinese participants while thinking about either their own traits, their mother's traits or a politician's traits. They found that while Western participants showed vmPFC activation only when thinking about themselves, Chinese participants, whose culture emphasises the interdependent nature of the self, showed mPFC activation in both the self and mother conditions suggesting that their brains were processing their mother's traits as self-relevant. A follow-up study showed that when students from Hong Kong, who can be considered bicultural due to the long period of British rule, are primed with Western identities they showed increased vmPFC differentiation between self and mother compared to when they were primed with Chinese identities (Ng, Han, Mao, \& Lai, 2010). Similar modulations of vmPFC activity have also be seen between Chinese students in America who have become less interdependent since arriving compared to those who became more interdependent (Chen, Wagner, Kelley, \& Heatherton, 2015). In addition, Chiao et al. (2009) showed that across cultures those with a more independent view of the self showed greater $\mathrm{mPFC}$ when thinking about general self-traits, e.g. "I am truthful", while those with an interdependent view of the self showed greater mPFC when thinking about contextual self-traits, e.g. "When talking to my mother I am truthful". The vmPFC has also been shown to have greater activation to objects owned by the participant (Kim \& Johnson, 2012), suggesting a role in representing an extended "material" self as well as an extended social self. 
In addition to holding information about self-relevant traits, preferences, etc., the conceptual self is also thought to strongly rely on autobiographical information stored in episodic memory. Meta-analyses of imaging studies have shown that autobiographical memory tasks recruit similar vmPFC areas to self-trait processing along with a number of other brain areas including the hippocampus (Spreng, Mar, \& Kim, 2009; Svoboda, McKinnon, \& Levine, 2006). Furthermore, a recent study examined functional connectivity between the hippocampus and neocortex during the retrieval and elaboration of episodic memories and found that while the dmPFC is functionally coupled to the hippocampus during the retrieval of memories the vmPFC strongly coupled to it both during retrieval and when participants are required to relive the memory in elaborate detail (McCormick, St-Laurent, Ty, Valiante, \& McAndrews, 2015). These results further demonstrate the importance of the vmPFC to the processing and experience of the conceptual self.

\section{Integrating the Bodily and Conceptual Self}

Until recently, there have been few attempts to consider the relationship between the bodily and conceptual self, or how the brain's representations of the bodily and conceptual selves interact. Farmer and Tsakiris (2012) suggested one possible branch between the two forms of selfhood by outlining the concept of a bodily social self, which is the first form of selfhood in which one's self is represented as an object of others' perceptions. The development of the bodily social self is closely entangled with the ability to represent others as having their own perspective, separate from one's own. This form of selfhood is also still considered ultimately bodily as, at least in its developmentally primitive form, it is thought to first rely upon recognising the commonality of one's own body to those of others. A similar tripartite distinction has been made by Sugiura (2013) who distinguishes between three different self-schemas: the physical self-schema which roughly corresponds to the bodily self described here; the interpersonal self-schema which emerges due to social interaction and processes how the self is perceived by other individuals; and the social-value self-schema which processes a more general sense of the self relative to others and is involved in the ascription of traits and social roles to the self. This social-value self roughly corresponds to the conceptual self as discussed in this article. Importantly, both of these two models of the self allow for cross-talk between the different forms of self-representation, which can allow changes in one form of self-representation to affect changes at another level in a hierarchical manner (see Feinberg, 2011 for an additional tripartite hierarchical model of the self).

In terms of neural links between bodily and conceptual self-representation, so far there have been few direct attempts to understand how the neural regions involved in the bodily self and the conceptual self influence each other. However, one recent meta-analysis of fMRI studies sought to identify common regions activated both when participants perceived their own face and when the saw self-relevant traits $(\mathrm{Hu}$ et al., 2016). The authors found that the only regions to show an overlap in activation across both tasks were the dorsal anterior cingulate and the vAI, suggesting that these regions may play a key role in specifying self-relevance across 
different domains. The finding of vAI activation is of particular significance given its role in processing interoceptive signals (Craig, 2009) and the role of the AI more generally in the processing of body ownership (Ehrsson et al., 2004; Limanowski et al., 2014). It should be noted, however, that the sense of bodily self used by $\mathrm{Hu}$ et al. related to the recognition of one's own face from the outside and as such significantly differed from the sense of the bodily self described above which is grounded in the ongoing multisensory perception of bodily signals from both interceptive and exteroceptive senses rather than mere recognition of visual representations of the self. As such, it is still unclear whether there is any specific brain area that is directly involved in processing all self-related information, or if the different types of neural self-representation interact with each other through connections between distinct networks.

\section{The Self and Prejudice}

Now that we have discussed the cognitive and neural bases, we will briefly highlight the strong interconnections between both bodily and conceptual self-representation and our social interactions with others. While the interconnections between the self and society are numerous and diverse, here we will focus on the relationship between the self and prejudice.

\section{Prejudice and the Conceptual Self}

Work on the relationship between the conceptual self and prejudice has a long pedigree. Tajfel $(1974,1982)$ emphasised the important role that in-group/out-group distinctions played in how individuals perceived themselves. He suggested that identifying with a larger group plays a key role in establishing an individual's identity and self-worth and further that this leads the in-group to be strongly associated with the self such that threats to the group are perceived as threating the individual's own self-concept. In support of this view, work on the formation of minimal groups shows that participants tend to show an in-group bias even for social groups with little meaningful distinctions between them and further that people tend to use inferences about the self to anchor their judgements about the ingroup (Otten, 2003). This process of in-group identification is highly likely to lead to prejudice as individuals will favour their own group and in some cases disparage the out-group in order to increase their sense of self-worth (Chang, Hodgins, Lin, \& Huang, 2014; Mackie \& Smith, 2002).

Empirical support for this position has come from a series of studies investigating the role of self-esteem and self-threat on intergroup prejudice. A series of studies have demonstrated that threats to the self either via reminder of mortality (Burke, Martens, \& Faucher, 2010; Maj \& Kossowska, 2015) or through negative feedback about oneself (Allen \& Sherman, 2011; Fein \& Spencer, 1997; Spencer, Fein, Wolfe, Fong, \& Duinn, 1998) can lead participants to increase their reliance on negative stereotypes about others. Allen and Sherman demonstrated that this effect was due to a greater focus on the negative attributes of out-groups, suggesting that 
these effects do represent a means of self-protection through the devaluation of nonself groups. Interestingly Rudman, Dohn and Fairchild (2007) showed that this process of self-enhancement could lead to prejudice even when the original threat to the self was that they displayed prejudice leading to "ironic racism". The authors also demonstrated that a process of self-affirmation following threat to self prevented the increase in intergroup prejudice by removing the need to protect selfworth.

In contrast to the role of self-threat on out-group prejudice, research has suggested that trait self-esteem has little bearing on prejudice towards other groups, although there is some evidence for differences in prejudice between high and low self-esteem participants expression of prejudice depending on whether in-group bias is measured explicitly or implicitly (Aberson, Healy, \& Romero, 2000). However, further research has distinguished between two types of self-esteem: explicit, i.e. what people report their self-esteem to be, and implicit, i.e. self-representations that operate largely outside conscious awareness (Greenwald \& Banaji, 1995). Jordan, Spencer and Zanna (2005) found that participants with high explicit but low implicit self-esteem showed increased prejudice against out-groups after self-threat, whereas those with high explicit and implicit self-esteem did not. This indicated that those with high implicit self-esteem either have a greater ability to suppress the impulse to derogate others or that they have greater internal self-affirmation so do not need to derogate others in order to enhance their self-worth.

How people conceptualise themselves has also been shown to affect the way they respond to prejudice from others. McCoy and Major (2003) found that women who identified strongly with their gender showed more signs of depressed affect and greater reductions in self-esteem when receiving a negative evaluation based on sexist stereotypes, as compared to women who did not identify strongly with their gender. Similarly, among Latino-Americans, group identification was positively correlated with depressed affect when reading about discrimination to an in-group member, but negatively related to depressed affect when reading about discrimination towards an ethnic out-group. However, recent work has exposed a more complex picture by demonstrating that while personally experiencing racial discrimination decreases self-esteem, the perception of increased discrimination against other members of one's ethnic group can lead to enhanced self-esteem (Armenta \& Hunt, 2009). This appears to occur through a process by which greater perception of group discrimination leads to greater identification with the in-group which in turn bolsters self-identity and self-worth.

The final source of evidence for the importance of the conceptual self to research on prejudice comes from neuroimaging studies of the brain basis of prejudice that highlight an important role for two key self-related areas, the vmPFC and the AI (Amodio, 2014). As discussed above, the vmPFC is heavily implicated in the representation of the conceptual self. Therefore, it is perhaps unsurprising that it is also sensitive to prejudice against other social groups. fMRI studies have demonstrated attenuated vmPFC activation when observing members of social groups that elicit feelings of disgust, e.g. homeless people and drug addicts (Harris $\&$ Fiske, 2007), and when men with sexist attitudes observe sexualised images of women (but not men) (Cikara, Eberhardt, \& Fiske, 2011). Furthermore, a recent 
study used transcranial direct current stimulation to increase activation in the mPFC while participants carried out a task measuring implicit racial bias and showed that stimulation of this region led to a decrease in racial bias (Sellaro et al., 2015).

In contrast, both the vAI and dAI have been found to show increased activation for out-groups compared to in-groups (Richeson et al., 2003; Ronquillo et al., 2007). While both AI regions have been strongly implicated in self-processing, their role as a centre for interoceptive information processing means that they are also linked to the visceral representation of emotion and particularly negative affect (Nitschke, Sarinopoulos, MacKiewicz, Schaefer, \& Davidson, 2006). Thus, it has been suggested that AI activation in prejudice studies represents the negative subjective feelings that participants experience when perceiving an out-group (Harris \& Fiske, 2006; Knutson, Mah, Manly, \& Grafman, 2007).

\section{Prejudice and the Bodily Self}

While the role of the conceptual self in prejudice has received a considerable amount of attention over the years, the role of the bodily self in social cognition has only recently begun to be appreciated. The key spur towards interest in this area was the discovery of "mirror neurons" in the macaque premotor and parietal cortices. These neurons fired both when the monkey performed an action, and when they observed the actions of another, suggesting a link between executing one's own movements and understanding the actions of others (di Pellegrino, Fadiga, Fogassi, Gallese, \& Rizzolatti, 1992; Gallese, Fadiga, Fogassi, \& Rizzolatti, 1996). Neuroimaging studies (Kilner, Neal, Weiskopf, Friston, \& Frith, 2009; Molenberghs, Cunnington, \& Mattingley, 2012) and more recently single-cell recordings (Mukamel, Ekstrom, Kaplan, Iacoboni, \& Fried, 2010) have demonstrated similar activations in humans.

Further research has suggested that similar systems of shared representation of self and other exist for perception of pain (Bernhardt \& Singer, 2012; Fitzgibbon, Giummarra, Georgiou-Karistianis, Enticott, \& Bradshaw, 2010), touch (Keysers, Kaas, \& Gazzola, 2010; Schaefer, Heinze, \& Rotte, 2012) and emotional processing (Bastiaansen, Thioux, \& Keysers, 2009; Jabbi, Bastiaansen, \& Keysers, 2008). While there is still considerable debate about exactly how these shared activations contribute towards the understanding of others (see the following reviews for three distinct accounts: Cook, Bird, Catmur, Press, \& Heyes, 2014; Gallese, 2007; Kilner, Friston, \& Frith, 2007), the involvement of neural systems involved in representing the bodily self in social cognition is now well established.

These shared regions for the processing of the actions and sensations of one's own body and that of others have also been shown to be sensitive to a number of different social factors including prejudice. In the motor domain, Gutsell and Inzlicht (2010) tested White participants and found less mu suppression, a sign of motor cortex activation, when viewing actions performed by a member of a racial out-group compared to when viewing those of a White person. Moreover, the amount of mu suppression attenuation was modulated by the prejudice of the participant. Similar results have been found using transcranial magnetic stimulation (TMS) (Désy \& Théoret, 2007; Molnar-Szakacs, Wu, Robles, \& Iacoboni, 2007) 
and fMRI (Liew, Han, \& Aziz-Zadeh, 2011). Interestingly, a follow-up study on White participants found that differences in mu suppression for Black versus White actors were abolished when the actors made a threatening action (Gutsell \& Inzlicht, 2013). Similarly, Rauchbauer, Majdandžić, Stieger, and Lamm (2016) showed that when White participants viewed an angry Black face while observing gestures, they showed greater interference in executing their actions than seeing an angry White face. These findings suggest that for White participants, threatening actions of Black individuals are given greater priority in processing. This may be a result of outgroup faces being perceived as more threatening, and so more important to predict and rapidly react to (Farmer, Apps, \& Tsakiris, 2016).

Moving from the motor to the sensory domain, prejudice has been shown to have particularly strong effects on the processing of other's pain. $\mathrm{Xu}, \mathrm{Zuo}$, Wang, and Han (2009) demonstrated that when participants observed painful stimuli being applied to members of their own racial group, they showed increased activation in both the anterior cingulate cortex (ACC) and the vAI, both areas known to be involved in processing one's own pain. However, when observing members of a different racial group receiving pain, activation in both these areas was significantly attenuated. Further research has shown that this attenuation of empathy for pain in out-group members is modulated both by implicit racial bias (Avenanti, Sirigu, \& Aglioti, 2010; Azevedo et al., 2013) and perceived similarity with the other person (Azevedo et al., 2013).

Prejudice has also been shown to modulate the mirroring of touch and emotions. Serino, Giovagnoli, and Làdavas (2009) demonstrated this using a paradigm called visual remapping of touch, in which observing touch on another's face leads to greater accuracy in tactile perception on one's own face. They found that this effect was reduced when observing a face from a different racial group. They also found a similar reduction when people viewed the face of a politician from an opposing political party, demonstrating that the effect was based on social, rather than purely physical, self-similarity. Similar findings have been reported in emotional processing. Gutsell and Inzlicht (2012) found that while viewing members of a racial ingroup being sad led to similar neural activation as being sad oneself, this effect was reduced for members of a racial out-group. Again, this effect was mediated by prejudice towards the out-group, suggesting that social identification rather than purely physical similarity is the key factor in group-based modulations of shared representations.

In addition to its effect on the activation of shared bodily representations, such as touch, emotions and pain, social group membership has also been shown to affect bodily responses during social interactions. Müller et al. (2011) showed that White participants showed an interference effect when performing contrasting actions in tandem with White actor but not with a Black actor. Similarly, Sacheli and colleagues (2015) found that when interacting with an in-group but not an out-group avatar the actions of the other person interfered with people's own action execution and that this effect was mediated by participant's implicit racial bias. Further evidence of prejudice affecting bodily interaction with others come from studies of mimicry, the unconscious tendency we have to imitate the facial and bodily movements of others, which is thought to be a method of increasing affiliation 
(Chartrand \& Lakin, 2013; Hale \& Hamilton, 2016). This tendency to mimic another person is reduced when the person belongs to a social or racial out-group (Bourgeois \& Hess, 2008; Liebert et al., 1972; Losin, Iacoboni, Martin, Cross, \& Dapretto, 2012; Neely, Heckel, \& Leichtman, 1973; van der Schalk et al., 2011; Weisbuch \& Ambady, 2008), and the extent to which we mimic out-group members is negatively related to our implicit prejudice against them. For example, Yabar, Johnston, Miles and Peace (2006) found greater mimicry of a non-Christian confederate than of a Christian confederate among non-Christian participants. They also found that the amount of actions of the Christian that participants mimicked was positively correlated with their implicit attitudes towards Christians.

\section{Changing the Self to Change Social Attitudes}

In the previous section, we discussed the strong links between self-representations and prejudice. However, the role that self-representation can play in the establishment and manifestation of prejudice also suggests that changing selfrepresentations may be a viable means of reducing prejudice. In this final section, we will look at evidence for the malleability of both the conceptual and bodily self and how that malleability can be harnessed in the aims of reducing prejudice. We will conclude by considering how current developments in technology are making changes in self-representation both more immersive and accessible, and consider some of the opportunities and risks of such developments for reducing racial bias.

\section{Reducing Prejudice Through the Conceptual Self}

Most studies aiming to alter the self in order to reduce prejudice have focussed on the conceptual self. Lai et al. (2014) tested 17 different interventions for reducing racial bias and found that amount of self-involvement was a key predictor of success. Here, we will consider a number of different ways the conceptual self can be modified ranging from simple associative learning between the self and others, through changes in group identity or self-construal and finally changes in perspective taking.

\section{Associative Learning}

Perhaps the most abstract means for altering the conceptual self is the linking of the self with another through associative learning. In the last few years, there has been an increased interest in investigating how associating different stimuli with the self can change how that stimuli are processed. This research has established that simply training people to associate self-relevant words (i.e. their own name or the word "You") with particular shapes can lead to improved perceptual processing of those shapes (Sui, He, \& Humphreys, 2012; Sui \& Humphreys, 2015). Similar perceptual effects have also been found when the same items are associated with rewards, suggesting that the effect is due to the greater value people assign themselves compared to others (Sui et al., 2012). In line with this, it has been shown that 
associating the self with a particular soft drink led to more positive implicit attitudes towards that drink but that this effect is mediated by self-esteem, with the increase in attitude being greatest for those with high self-esteem and less for those with low self-esteem (Prestwich, Perugini, Hurling, \& Richetin, 2010). The neural basis of the effect of self-association on perceptual processing has been shown to be due to increased coupling between vmPFC, which as described above plays a key role in self-processing, and the left superior temporal sulcus, an area known to play an important role in directing attention to external stimuli (Sui, Rotshtein, \& Humphreys, 2013).

Similar effects of self-association have also been found for more socially relevant stimuli. Payne, Tsakiris, and Maister (2016) demonstrated that labelling the faces of others as being self-relevant in a brief learning task led to increased perceptual processing of the other's face as compared to non-self-associated faces. In addition, self-association has been shown to enhance visual perspective taking. Participants were faster to take the perspective of an avatar associated with the self than with non-self-relevant avatars and showed greater interference when their own perspective and that of the self-tagged avatar were in conflict (Mattan, Quinn, Apperly, Sui, \& Rotshtein, 2015).

\section{Group Membership and Self-Construal}

At present, there have been no published studies that sought to investigate whether this basic method of self-association can impact upon prejudice towards an outgroup. However, a more naturalistic way of eliciting a self-association with individuals of an out-group is to assign individuals to a "new" social group the definitions of which cut across existing social boundaries. Kurzban, Tooby, and Cosmides (2001) demonstrated that memory biases in favour of a racial in-group (White people) against a racial out-group (Black people) could be removed by four minutes of training that led to the association of some faces from each group as members of the same team as the participant with the effects previously associated with race now applying to the newly created in-group/out-group categories.

The creation of a new in-group has since been shown to modulate more direct measures of prejudice. For example, placing a participant in an arbitrary team with members of a racial out-group reduces racial prejudice against those members (Van Bavel \& Cunningham, 2009). Similarly, Sheng and Han (2012) found that including members of a racial out-group as a member of one's own team for competitions eliminated an in-group bias in participants' neural response to pained facial expressions. However, in both studies this effect did not appear to generalise to racial out-group members who were part of the opposing team, nor to those who were not members of either team, suggesting it has limited generalisability as a means of consistently reducing prejudice. A more powerful version of linking the self to out-group members was reported by (Lai et al., 2014). Here, non-Black participants played dodgeball on a team composed fully of Black players, against an all-White player team. This method was found to be highly effective at reducing racial prejudice. This suggests that to reduce prejudice in a generalised manner, it 
may be necessary to both raise self-identification with the out-group and reduce identification with the in-group.

Prejudice towards an out-group can also be altered by changing self-construal. Wang, Wu, Liu, Wu, and Han (2014) showed that priming Chinese participants with independent self-construal, which emphasises the self as an individual, as opposed to interdependent self-construal, which emphasises the self as situated with in a social group, led to decreased in-group bias in the neural response to pain in the mid-cingulate, supplementary motor area and AI. This suggests that conceptualising oneself as independent from one's own social group leads to a corresponding decrease in bias towards that group in empathic neural processing.

\section{Perspective Taking}

Although laboratory-based self-association procedures lack ecological validity, there is evidence to suggest that more naturalistic processes may elicit the formation of similar self-associations. One example of this is perspective taking, which is the process of imagining oneself in the position of another individual. Importantly, this process not only leads to a reduction in prejudice against others, but it appears to achieve this by creating a new association between the self and that other. A number of studies have demonstrated that taking the perspective of the member of a social out-group can lead to reduced in-group bias and more positive attitudes towards that group (Galinsky \& Ku, 2004; Galinsky, Ku, \& Wang, 2005; Shih, Wang, Trahan Bucher, \& Stotzer, 2009; Todd \& Burgmer, 2013; Vescio, Sechrist, \& Paolucci, 2003). Furthermore, Müller et al. (2011) found that an in-group bias in motor interference disappeared when White participants took the perspective of their Black interaction partner.

Crucially, there is now strong evidence that this change in attitudes is driven by an increase in self-association towards the other rather than alternative possibilities such as an increase in empathy for the other person. For example, Galinsky and $\mathrm{Ku}$ (2004) found that participants' own self-esteem mediated the change in social attitudes caused by perspective taking while Todd and Burgmer (2013) showed that the effects of perspective taking on improved social attitudes were diminished when participants were primed with negative self-related information. In addition, Marini, Rubichi, and Sartori (2012) found that imagining one's self being helped by an outgroup improved participant's attitudes towards that out-group whereas imaging another person being helped did not. Another recent study demonstrated that actively taking the perspective of a black character who experienced racial bias in a computer game led to a greater reduction in implicit racial bias than merely reading the about the same experience through text did (Gutierrez et al., 2014). It should be noted, however, that Lai et al. (2014) found no evidence that perspective taking of an out-group member led to reduced bias towards that out-group. This fact may be explained by the fact that in their study the method of perspective taking used was relatively basic consisting of simply seeing a person's face and a short sentence about them. In contrast, the more successful instances of perspective taking tend to involve more immersive techniques such as writing a short essay from the other's perspective (Galinsky \& Ku, 2004; Todd \& Burgmer, 2013), writing their views 
about a film clip featuring an out-group member (Shih et al., 2009) or taking the role of an out-group member in a computer game (Gutierrez et al., 2014).

The role of perspective taking in changing social attitudes may also explain findings that fiction about the experiences of members of other groups can lead to increased empathy and positive attitudes towards those groups (Bilali \& Vollhardt, 2013; Hakemulder, 2000, 2008; Johnson, Jasper, Griffin, \& Huffman, 2013; Paluck, 2007; Vezzali, Stathi, Giovannini, Capozza, \& Trifiletti, 2015). Several researchers have suggested that fiction has a functional role as a form of technology which allows us to experience the world through the eyes of others and thus allows us to identify more closely with them blurring the boundary between self and other (Koopman \& Hakemulder, 2015; Mar \& Oatley, 2008; Oatley, 2016). Indeed, some authors have argued that, through allowing people to emphasise with others of a different gender, class or nationality, the growth in literacy and explosion of literature during the eighteenth and nineteenth centuries played a key role in the development of the idea of universal human rights (Hunt, 2007; Pinker, 2011).

While the exact characteristics of fiction that produce increased positive attitudes and greater empathy towards others are difficult to disentangle due to its multifaceted nature, the act of associating with another via stepping into their role has been highlighted as particularly relevant (Koopman \& Hakemulder, 2015; Oatley, 2016). Given that many (e.g. Dennett, 1991; Goffman, 1959; Polkinghorne, 1991) have suggested that the conceptual self is itself reliant on our ability to craft narratives about our own lives, it is perhaps unsurprising the vicariously experiencing the life of another can lead to changes in self-identification.

\section{Reducing Prejudice Through the Bodily Self}

Having outlined how changing the conceptual self can alter social attitudes, we now turn to whether the malleability of the bodily self can also be harnessed to alter social views. Although the links between the bodily self and social processing are somewhat less clear than for the conceptual self, the past few years have seen increased interest in this question. Here, we will first consider two key ways by which altering the bodily self may influence social attitudes towards others, before moving on to discuss how technological innovations are making this topic increasingly relevant.

\section{Synchrony, Imitation and Social Interaction}

The first method of changing social attitudes through the bodily self comes from studies on the power of synchronous movement and mimicry to improve cooperation. Synchronous movement plays an important part in building social affiliation; many social situations that are known to increase affiliation involve synchronised movement in some way. Dancing is one of the clearest instances of social synchrony, and it has been argued that the human propensity for dance and music has emerged as the result of an adaptation to facilitate group bonding and the sense of being part of a tight-knit community (Brown, 2000; Levitin, 2008). Crowds at sporting events, religious gatherings and political rallies will often chant, sing and 
move in synchrony, Japanese corporations have long used communal exercise such as Tai Chi to encourage a sense of team work among their employees, and soldiers are trained to march in step in order to develop the feeling of being part of a cohesive unit. It has been suggested that the power of synchrony in social situations stems from the fact that synchronous action blurs the boundary between self and other, causing the sense of bodily selfhood to expand to include the other (Farmer \& Tsakiris, 2012; Pacherie, 2014).

Experimental studies have demonstrated that moving in synchrony with others has a significant effect on people's reported feelings towards interaction partners and leads to the building of affiliation (Hove \& Risen, 2009), increased attraction (Nowak, Watt, \& Walther, 2005) and compassion (Valdesolo \& Desteno, 2011). A number of other studies have used techniques from behavioural economics to investigate to what extent synchrony can affect people's actual behaviour. For example, both walking and singing in synchrony with a group of others lead to greater group cooperation in economic games (Wiltermuth \& Heath, 2009). In addition, Launay, Dean, and Bailes (2013) found that simply seeing another person tapping in time with one's actions is enough to increase monetary investments in a trust game. Fischer, Callander, Reddish, and Bulbulia (2013) used an even more ecologically valid approach by having participants take part in real religious rituals that involved varying degrees of synchronised movement. They showed that the amount of synchrony involved in a ritual was a key factor in increasing the amount of money offered by participants in a subsequent public goods game. The same authors also found that the effect of synchronous action on pro-social behaviour was not confined to interaction partners but also encompassed non-partners, indicating that synchronous action can act as a general prime towards pro-social behaviour (Reddish, Bulbulia, \& Fischer, 2014). This last finding suggests that the effects of synchronous action with one member of a social out-group may generalise towards other out-group members.

At present, there have been relatively few studies investigating how synchrony affects attitudes towards other social groups. However, one recent study by Tunçgenç \& Cohen (2016) found that synchronous movements between two minimally defined groups of children lead to greater bonding between the groups than did asynchronous movements. However, other studies have examined how the closely related concept of imitation can change attitudes towards more established social out-groups. Inzlicht, Gutsell, and Legault (2012) found that after imitating the actions of a Black model, White participants showed more positive implicit attitudes towards Black people. This was not the case when the participants imitated a White model or when they simply watched the actions of a Black model, suggesting that both exposure to the out-group and similarity between the movements of self and other in are necessary for changing attitudes. There is also evidence that being mimicked by an out-group can improve social attitudes. In a study by Hasler, Hirschberger, Shani-Sherman, and Friedman (2014), Israeli participants discuss the issue of the wall between Israel and Palestine with a virtual avatar of a Palestinian whose postural movements were either the same (mimicry) or the opposite (nonmimicry) of their own. They found that participants who were mimicked showed increased positive affect towards their interaction partners and greater empathy with 
the situation of the Palestinians more generally, as assessed by their responses during the interaction. However, mimicry did not affect their explicit feeling towards Palestinians as a whole, suggesting that further work is required to determine whether mimicry by one out-group member leads to improved attitudes for the whole group.

\section{Embodiment}

While similarity of bodily movements during social interaction suggests one naturalistic way to alter the relationship between self and others on a bodily level, recent scientific and technological developments are allowing us to go further by changing the direct perception of our own body compared to others. In this section, we will outline the evidence from studies using virtual reality (VR) and bodily illusions showing that we can successfully embody out-group members, before moving on to discuss the opportunities and challenges that the growing availability of VR technology offers for changing social attitudes.

Over the last decade, there has been increased interest in how multisensoryinduced changes in body representations can lead to changes in social attitudes. The earliest work in this area was a study by Yee \& Bailenson (2006) in which participants wore a head mounted display (HMD) which placed them in a virtual body while tracking their head movements, creating a sense of visual-motor synchrony that allowed for the experience of ownership of their virtual avatar. The study found that when participants were given an avatar with an elderly face they subsequently showed reduced negative stereotyping of the elderly. However, this study did not test for effects of embodiment on implicit bias, and many of the participants guessed the purpose of the study leaving open the possibility that the findings were driven by demand characteristics (McCambridge, de Bruin, \& Witton, 2012).

More recent work has sought to test the effect of embodiment on prejudice in a more implicit manner. In a series of studies, the authors of the current paper used the rubber hand illusion (RHI) to show that synchronous visual-tactile stimulation over a Black hand decreased implicit racial bias towards Black people in White participants (Farmer, Maister, \& Tsakiris, 2014; Farmer, Tajadura-Jiménez, \& Tsakiris, 2012; Maister, Sebanz, Knoblich, \& Tsakiris, 2013). Importantly, two of these studies (Farmer et al., 2012; Maister et al., 2013) found that the amount that participant's racial bias decreased was correlated with the strength of the ownership they felt for the hand, suggesting that this effect is partly caused by the increased overlap between the self and the racial out-group. Effects of embodiment on racial bias have also been found when using VR to generate a full-body illusion (Peck, Seinfeld, Aglioti, \& Slater, 2013) and similar changes in implicit attitudes to children have been found after embodying a child avatar (Banakou, Groten, \& Slater, 2013). However, another recent study (Estudillo \& Bindemann, 2016) found no evidence that multisensory stimulation of the face led to changes in racial bias. This may be due to the fact that, unlike the RHI or the full-body illusion, visualtactile stimulation to the face does not produce a strong subjective feeling of 
embodiment, probably due to the greater distinctiveness of facial features compared to representations of hands or even body shape.

In addition to these effects on implicit bias, multisensory stimulation has also been shown to affect more low-level sensorimotor measures of prejudice. Fini, Cardini, Tajadura-Jiménez, Serino, and Tsakiris (2013) found that synchronous touch on one's own face and that of a racial out-group member served to eliminate in-group bias in the extent to which we "resonate" with other people's tactile experiences (the "visual remapping of touch" effect). Furthermore, this change was mediated by participant's pre-existing implicit racial bias, with the greatest change occurring for those with the most bias. In another example of self-other merging, Maister, Cardini, Zamariola, Serino, and Tsakiris (2015) showed that multisensory stimulation of one's own face and another led to a remapping of one's own peripersonal space onto the body of the other. Another recent study combined research on embodiment and imitation by showing that White participants embodying a Black avatar showed a greater tendency to imitate the posture and movements of a Black virtual character than a White one while the opposite applied when they embodied a White avatar (Hasler, Spanlang, \& Slater, 2017). These examples highlight the role that embodiment can play in blurring the boundaries between self and other and its value in changing both attitudes and more low-level bodily responses towards other social groups and individuals.

\section{Future Directions: Changing Attitudes via Embodiment Outside the Laboratory}

Having considered the current evidence for the effectiveness of embodiment in changing social attitudes, we now wish to consider how these findings might be harnessed in order to help reduce prejudice outside of the laboratory, and also highlight possible pitfalls that such efforts will have to overcome. The past year has seen the launch of several commercial VR systems such as the Oculus Rift, the HTC Vive and the Samsung Gear which are capable of supporting the visual-motor synchrony necessary to allow the experience of embodiment.

There is now considerable interest from game designers, artists and journalists about how such technology could be used to better immerse people in virtual worlds and increase their empathy with others. For example, the BBC recently released a $360^{\circ} 3 \mathrm{D}$ video from the Calais migrant camp which aimed to give viewers a more full and immersive view of the conditions that residents of the camp must endure (BBC, 2016). Another example is "The Machine to Be Another", which is an innovative collaboration between scientists and artists in Barcelona which has used the body-swap illusion developed by Petkova and Ehrsson (2008) to allow users to experience what it is like to have the body of another gender, a dancer or a person with disabilities (Be Another Lab, 2016; Souppouris, 2014). VR embodiment has also been applied in clinical settings and is being used in the treatment for psychiatric conditions (Riva, Banos, Botella, Mantovani, \& Gaggioli, 2016; Valmaggia, Latif, Kempton, \& Rus-Calafell, 2016) and stroke rehabilitation (Iruthayarajah, McIntyre, Cotoi, Macaluso, \& Teasell, 2016; Teo et al., 2016). 
Given the relative novelty of VR, there are only a limited number of studies that have been conducted on its effects. Therefore, there are many questions that need to be answered empirically before the opportunities and risks of VR can be truly appreciated. For example, at present it is unknown how long the effect of embodiment on prejudice reduction lasts. The studies mentioned in the previous section all measured the effects of embodiment on implicit attitudes immediately after the end of the embodiment session, and whether such interventions lead to long-term changes in attitudes remains to be seen. The neural basis of embodiment's power to change social attitudes is also currently unknown, and future research should aim to ascertain how these changes at the level of the bodily self are able to lead to changes in brain areas involved in the processing of higher-level conceptual self-representations such as the vmPFC.

For the moment, however, it is possible to extrapolate what we know from nonVR computer games in order to make some predictions regarding the effects of VR embodiment on prejudice reduction and empathy. Although the avatars that we control in standard computer games are not fully embodied, they are tied to the self via association and have been relatively well studied by cognitive scientists. Several studies have shown that taking another's identity in a computer game leads to more positive attitudes towards that other, via a generalisation of positive selfassociations to the other (Blake, Hefner, Roth, Klimmt, \& Vorderer, 2012; Lewis, Weber, \& Bowman, 2008; Li, Liau, \& Khoo, 2013). Importantly, this generalisation of associations between self and other during computer game play is not unidirectional; in fact, there is considerable evidence that taking on the identity of another leads to changes in self-processing based on our beliefs and stereotypes about that other, a phenomena known as the Proteus effect (Yee \& Bailenson, 2007; Yee, Bailenson, \& Ducheneaut, 2009).

The Proteus effect has been shown to manifest under various different conditions. Yee and Bailenson (2007) found that giving people an attractive avatar led them to be more open and intimate with a confederate, while giving them a taller avatar led them to behave more confidently in a negotiating task. Similarly, having an obese avatar has been shown to lead men to expend less exercise during a computer based sports game (Peña, Khan, \& Alexopoulos, 2016) and controlling an avatar dressed as a member of the $\mathrm{Ku}$ Klux Klan lead people to create more aggressive and less affiliative narratives than controlling an avatar dressed as a doctor (Peña, Hancock, \& Merola, 2009). Other studies have shown that the Proteus effect also applies to fully embodied VR; Freeman et al. (2014) showed that embodying a short avatar led to more negative self-evaluation and greater feelings or paranoia, while Rosenberg, Baughman, and Bailenson (2013) showed that, after embodying an avatar with the superhero-like power of flight, participants displayed greater pro-social behaviour. Interestingly, although the Proteus effect was designed to explain the effects of using virtual avatars in computer games, there is evidence that any cognitive processing that activates stereotypes about others can lead to stereotypes about the other being transferred onto the self (DeMarree, Wheeler, \& Petty, 2005; Kawakami et al., 2012; Wheeler, Demarree, \& Petty, 2007).

The existence of the Proteus effect suggests that in some cases embodying an avatar of a disadvantaged social group may have the counter-productive effect of 
activating stereotypes associated with that group rather than breaking them down. For example, it has been shown that participants of either gender showed greater mathematical problem solving when assigned to a male avatar competing against female avatars than did those assigned a female avatar competing against male avatars, suggesting that the Proteus effect worked to increase stereotypes about gender differences in mathematics (Lee, Nass, \& Bailenson, 2013). It has also been found that assigning female participants to sexualised avatars increased their selfobjectification and made them more likely to blame victims of rape for their situation (Fox, Ralston, Cooper, \& Jones, 2014). Others studies have shown that playing a Black character in a computer game leads participants to show more aggressive cognition and affect, presumably as a result of the activation of stereotypical views of Black men as violent (Ash, 2016; Eastin, Appiah, \& Cicchirllo, 2009). Importantly, Ash (2016) demonstrated that this effect was mediated by the extent to which users felt embodied in their avatar, suggesting that increased use of VR-based embodiment in gaming could lead to increased reinforcement of stereotyped views.

Currently, there has been little research on the Proteus effect and prejudice in fully embodied VR. However, two relevant studies have been conducted that suggest that it may also affect full embodiment. Groom, Bailenson, and Nass (2009) examined the effect of embodiment in a Black avatar during a job interview scenario, a situation associated with racial discrimination (Dasgupta, 2004), and found that participants subsequently showed increased rather than decreased racial bias. In another study, Kilteni, Bergstrom, and Slater (2013) found that participants embodying a black avatar exhibited higher variation and frequency of movement in a drumming task than did those embodying a white avatar; a finding the authors suggested was due to the stereotype of Black people as more rhythmic. Further research on the question of how much these divergent effects of embodying an outgroup on prejudice towards that group should examine the role of context in such manipulation. For example, researchers could have participants embody female or black avatars that are placed in either stereotypical or counter-stereotypical settings (e.g. a woman either cooking at home or working as a scientist in a laboratory) and then investigate the relationship between type of avatar embodied, setting and strength of embodiment on participant's implicit associations towards the embodied group.

These examples of how the Proteus effect can lead to the activation of stereotypes during embodiment point to the importance of being sensitive to the context in which the embodiment takes place, in order to ensure successful reduction of prejudice. This point is of particular relevance considering that the most widespread use of embodiment in the future is likely to be in the form of video games. Several recent reviews have demonstrated that both women and non-White ethnic groups are under-represented in video games, and when they are featured, they tend to be depicted in highly stereotypical and negative ways (Burgess, Dill, Stermer, Burgess, \& Brown, 2011; Deskins, 2013; Dickerman, Christensen, \& KerlMcClain, 2008). Furthermore, there is evidence that mere exposure to, as opposed to identification with, depictions of women and non-White ethnicities in video games can lead to negative attitudes towards those groups (Dill, Brown, \& Collins, 2008; 
Dill \& Burgess, 2012). Given the evidence that embodiment can lead to a stronger activation of stereotypes than other forms of media (Ash, 2016; Groom et al., 2009), it is important that the portrayal of women, Black, Asian and minority ethnic (BAME) people and members of other disadvantaged social groups is treated as an important issue by game designers. As VR technology advances to allow increased malleability of the boundaries between self and other, it is vital that those involved in designing the new forms of media and entertainment that make use of VR are aware of the need to combat lazy and stereotypic depictions of other social groups, and instead use these advances to present members of other group in a manner that builds up empathy and reduces prejudice between groups.

\section{Conclusion}

This paper has explored how prejudice and negative attitudes towards different groups can be altered by changing their closeness to the self. We have described an important division between two forms of selfhood, the more basic "bodily self" and the more abstract and socially constructed "conceptual self". We then examined the role of the self in prejudice, showing that both the bodily and the conceptual self are sensitive to group differences. The self can be involved in the construction of ingroup/out-group distinctions, and in the derogation of disliked social groups. In addition, we highlighted the strong links between brain regions involved in selfrepresentation are also involved in the social cognitive processes that underlie prejudice. We then moved on to presented evidence that the malleable nature of self-representations, both at the conceptual and bodily levels, offers a powerful method of changing social attitudes and reducing prejudice. Finally, we considered how the increasing availability of VR, which allows for the experience of fully embodying another person, presents opportunities and hazards for approaches that aim to use the self to change social attitudes. In summary, we present evidence for the importance of considering the role of the self in the development and maintenance of prejudice as well as suggesting that the malleability of selfrepresentation opens up several important new directions and issues for those interested in prejudice reduction.

Open Access This article is distributed under the terms of the Creative Commons Attribution 4.0 International License (http://creativecommons.org/licenses/by/4.0/), which permits unrestricted use, distribution, and reproduction in any medium, provided you give appropriate credit to the original author(s) and the source, provide a link to the Creative Commons license, and indicate if changes were made.

\section{References}

Aberson, C. L., Healy, M., \& Romero, V. (2000). Ingroup bias and self-esteem: A meta-analysis. Personality and Social Psychology Review, 4(2), 157-173.

Allen, T. J., \& Sherman, J. W. (2011). Ego threat and intergroup bias: A test of motivated-activation versus self-regulatory accounts. Psychological Science, 22(3), 331-333. 
Amodio, D. M. (2014). The neuroscience of prejudice and stereotyping. Nature Reviews Neuroscience, 15(10), 670-682.

Apps, M. A. J., Tajadura-Jiménez, A., Sereno, M. I., Blanke, O., \& Tsakiris, M. (2015). Plasticity in unimodal and multimodal brain areas reflects multisensory changes in self-face identification. Cerebral Cortex, 25(1), 46-55.

Apps, M. A. J., \& Tsakiris, M. (2014). The free-energy self: A predictive coding account of selfrecognition. Neuroscience and Biobehavioral Reviews, 41, 85-97.

Araujo, H. F., Kaplan, J. T., \& Damasio, A. (2013). Cortical midline structures and autobiographical-self processes: An activation-likelihood estimation meta-analysis. Frontiers in Human Neuroscience, 7, 548.

Armenta, B. E., \& Hunt, J. S. (2009). Responding to societal devaluation: Effects of perceived personal and group discrimination on the ethnic group identification and personal self-esteem of Latino/ Latina adolescents. Group Processes \& Intergroup Relations, 12(1), 23-39.

Ash, E. M. (2016). Priming or proteus effect? Examining the effects of avatar race on in-game behavior and post-play aggressive cognition and affect in video games. Games and Culture, 11(4), 422-440.

Avenanti, A., Sirigu, A., \& Aglioti, S. M. (2010). Racial bias reduces empathic sensorimotor resonance with other-race pain. Current Biology, 20(11), 1018-1022.

Azevedo, R. T., Macaluso, E., Avenanti, A., Santangelo, V., Cazzato, V., \& Aglioti, S. M. (2013). Their pain is not our pain: Brain and autonomic correlates of empathic resonance with the pain of same and different race individuals. Human Brain Mapping, 34(12), 1-14.

Banakou, D., Groten, R., \& Slater, M. (2013). Illusory ownership of a virtual child body causes overestimation of object sizes and implicit attitude changes. Proceedings of the National Academy of Sciences, 110(31), 12846-12851.

Bastiaansen, J. A. C. J., Thioux, M., \& Keysers, C. (2009). Evidence for mirror systems in emotions. Philosophical Transactions of the Royal Society B: Biological Sciences, 364(1528), 2391-2404.

BBC. (2016). Calais migrants VR. Retrieved October 10, 2016, from http://www.bbc.co.uk/taster/ projects/calais-migrants-vr

Be Another Lab. (2016). The machine to be another. Retrieved October 12, 2016, from http://www. themachinetobeanother.org/

Bekrater-Bodmann, R., Foell, J., Diers, M., Kamping, S., Rance, M., Kirsch, P., et al. (2014). The importance of synchrony and temporal order of visual and tactile input for illusory limb ownership experiences-An fMRI study applying virtual reality. PLOS ONE, 9(1), e87013.

Bermúdez, J. L. (1998). The paradox of self-consciousness. Cambridge, MA: MIT Press.

Bernhardt, B. C., \& Singer, T. (2012). The neural basis of empathy. Annual Review of Neuroscience, 35, $1-23$.

Bilali, R., \& Vollhardt, J. R. (2013). Priming effects of a reconciliation radio drama on historical perspective-taking in the aftermath of mass violence in Rwanda. Journal of Experimental Social Psychology, 49(1), 144-151.

Blake, C., Hefner, D., Roth, C., Klimmt, C., \& Vorderer, P. (2012). Cognitive processes involved in video game identification. In M. Herrlich, R. Malaka, \& M. Masuch (Eds.), Entertainment computingICEC 2012: 11th International Conference, Bremen, Germany, Proceedings (pp. 75-84). Berlin, Heidelberg: Springer.

Botvinick, M. M., \& Cohen, J. (1998). Rubber hands "feel" touch that eyes see. Nature, 391, 756.

Bourgeois, P., \& Hess, U. (2008). The impact of social context on mimicry. Biological Psychology, 77(3), $343-352$.

Brown, S. (2000). The "musilanguage" model of music evolution. In N. L. Wallin, B. Merker, \& S. Brown (Eds.), The origins of music (pp. 271-300). Cambridge, MA: MIT Press.

Burgess, M. C. R., Dill, K. E., Stermer, S. P., Burgess, S. R., \& Brown, B. P. (2011). Playing with prejudice: The prevalence and consequences of racial stereotypes in video games. Media Psychology, 14(3), 289-311.

Burke, B. L., Martens, A., \& Faucher, E. H. (2010). Two decades of terror management theory: A metaanalysis of mortality salience research. Personality and Social Psychology Review, 14(2), 155-195.

Capelari, E. D. P., Uribe, C., \& Brasil-Neto, J. P. (2009). Feeling pain in the rubber hand: Integration of visual, proprioceptive, and painful stimuli. Perception, 38(1), 92-99.

Carter, R. M., \& Huettel, S. A. (2013). A nexus model of the temporal-parietal junction. Trends in Cognitive Sciences, 17(7), 328-336. 
Chang, J. H., Hodgins, H. S., Lin, Y. C., \& Huang, C. L. (2014). On the flexibility of the automatic UsThem divide. In N. Weinstein (Ed.), Human motivation and interpersonal relationships: Theory, research, and applications (pp. 97-119). Dordrecht: Springer.

Chang, L. J., Yarkoni, T., Khaw, M. W., \& Sanfey, A. G. (2013). Decoding the role of the insula in human cognition: Functional parcellation and large-scale reverse inference. Cerebral Cortex, 23(3), 739-749.

Charlesworth, L. A., Allen, R. J., \& Havelka, J. (2016). Who am I? Autobiographical retrieval improves access to self-concepts. Memory, 4(8), 1033-1041.

Chartrand, T. L., \& Lakin, J. L. (2013). The antecedents and consequences of human behavioral mimicry. Annual Review of Psychology, 64, 285-308.

Chen, P.-H. A., Wagner, D. D., Kelley, W. M., \& Heatherton, T. F. (2015). Activity in cortical midline structures is modulated by self-construal changes during acculturation. Culture and Brain, 3(1), 39-52.

Chiao, J. Y., Harada, T., Komeda, H., Li, Z., Mano, Y., Saito, D. N., et al. (2009). Neural basis of individualistic and collectivistic views of self. Human Brain Mapping, 30(9), 2813-2820.

Cikara, M., Eberhardt, J. L., \& Fiske, S. T. (2011). From agents to objects: Sexist attitudes and neural responses to sexualized targets. Journal of Cognitive Neuroscience, 23(3), 540-551.

Cohen, D., Leung, A. K. A. K., \& Hoshino-Browne, E. (2007). Culture and the structure of personal experience: Insider and outsider phenomenologies of the self and social world. Advances in Experimental Social Psychology, 39(6), 1-67.

Cook, R., Bird, G., Catmur, C., Press, C., \& Heyes, C. M. (2014). Mirror neurons: From origin to function. Behavioral and Brain Sciences, 37(2), 177-241.

Craig, A. D. B. (2003). Interoception: The sense of the physiological condition of the body. Current Opinion in Neurobiology, 13(4), 500-505.

Craig, A. D. B. (2009). How do you feel-now? The anterior insula and human awareness. Nature Reviews Neuroscience, 10(1), 59-70.

Damasio, A. (1999). The feeling of what happens: Body, emotion and the making of consciousness. London: Vintage.

Dasgupta, N. (2004). Implicit ingroup favoritism, outgroup favoritism, and their behavioral manifestations. Social Justice Research, 17(2), 143-169.

De Vignemont, F. (2014). A multimodal conception of bodily awareness. Mind, 123(492), 989-1020.

Deen, B., Pitskel, N. B., \& Pelphrey, K. A. (2011). Three systems of insular functional connectivity identified with cluster analysis. Cerebral Cortex, 21(7), 1498-1506.

DeMarree, K. G., Wheeler, S. C., \& Petty, R. E. (2005). Priming a new identity: Self-monitoring moderates the effect of nonself primes on self-judgments and behavior. Journal of Personality and Social Psychology, 89(5), 657-671.

Dennett, D. C. (1991). Consciousness explained. Boston: Brown.

Denny, B. T., Kober, H., Wager, T. D., \& Ochsner, K. N. (2012). A meta-analysis of functional neuroimaging studies of self- and other judgments reveals a spatial gradient for mentalizing in medial prefrontal cortex. Journal of Cognitive Neuroscience, 24(8), 1742-1752.

Deskins, T. G. (2013). Stereotypes in video games and how they perpetuate prejudice. McNair Scholars Research Journal, 6(1), 19-36.

Désy, M.-C., \& Théoret, H. (2007). Modulation of motor cortex excitability by physical similarity with an observed hand action. PLoS ONE, 2(10), e971.

di Pellegrino, G., Fadiga, L., Fogassi, L., Gallese, V., \& Rizzolatti, G. (1992). Understanding motor events: A neurophysiological study. Experimental Brain Research, 91(1), 176-180.

Dickerman, C., Christensen, J., \& Kerl-McClain, S. B. (2008). Big breasts and bad guys: Depictions of gender and race in video games. Journal of Creativity in Mental Health, 3(1), 20-29.

Dill, K. E., Brown, B. P., \& Collins, M. A. (2008). Effects of exposure to sex-stereotyped video game characters on tolerance of sexual harassment. Journal of Experimental Social Psychology, 44(5), 1402-1408.

Dill, K. E., \& Burgess, M. C. R. (2012). Influence of black masculinity game exemplars on social judgments. Simulation \& Gaming, 44(4), 562-585.

Dummer, T., Picot-Annand, A., Neal, T., \& Moore, C. (2009). Movement and the rubber hand illusion. Perception, 38(2), 271-280.

Eastin, M. S., Appiah, O., \& Cicchirllo, V. (2009). Identification and the influence of cultural stereotyping on postvideogame play hostility. Human Communication Research, 35(3), 337-356. 
Eckert, M. A., Menon, V., Walczak, A., Ahlstrom, J., Denslow, S., Horwitz, A., et al. (2009). At the heart of the ventral attention system: The right anterior insula. Human Brain Mapping, 30(8), 2530-2541.

Edelman, G. M. (2004). Wider than the sky: The phenomenal gift of consciousness. London: Yale University Press.

Ehrsson, H. H. (2007). The experimental induction of out-of-body experiences. Science, 317, 1048.

Ehrsson, H. H., Spence, C., \& Passingham, R. E. (2004). That's my hand! Activity in premotor cortex reflects feeling of ownership of a limb. Science, 305(5685), 875-877.

Estudillo, A. J., \& Bindemann, M. (2016). Multisensory stimulation with other-race faces and the reduction of racial prejudice. Consciousness and Cognition, 42, 325-339.

Farmer, H., Apps, M. A. J., \& Tsakiris, M. (2016). Reputation in an economic game modulates premotor cortex activity during action observation. European Journal of Neuroscience, 44, 2191-2201.

Farmer, H., Maister, L., \& Tsakiris, M. (2014). Change my body, change my mind: The effects of illusory ownership of an outgroup hand on implicit attitudes toward that outgroup. Frontiers in Psychology, 4, 1016.

Farmer, H., Tajadura-Jiménez, A., \& Tsakiris, M. (2012). Beyond the colour of my skin: How skin colour affects the sense of body-ownership. Consciousness and Cognition, 21(3), 1242-1256.

Farmer, H., \& Tsakiris, M. (2012). The bodily social self: A link between phenomenal and narrative selfhood. Review of Philosophy and Psychology, 3(1), 125-144.

Fein, S., \& Spencer, S. J. (1997). Prejudice as self-image maintenance: Affirming the self through derogating others. Journal of Personality and Social Psychology, 73(1), 31-44.

Feinberg, T. E. (2011). The nested neural hierarchy and the self. Consciousness and Cognition, 20(1), 4-15.

Fini, C., Cardini, F., Tajadura-Jiménez, A., Serino, A., \& Tsakiris, M. (2013). Embodying an outgroup: The role of racial bias and the effect of multisensory processing in somatosensory remapping. Frontiers in Behavioral Neuroscience, 7, 165.

Fischer, R., Callander, R., Reddish, P., \& Bulbulia, J. (2013). How do rituals affect cooperation?: An experimental field study comparing nine ritual types. Human Nature, 24(2), 115-125.

Fitzgibbon, B. M., Giummarra, M. J., Georgiou-Karistianis, N., Enticott, P. G., \& Bradshaw, J. L. (2010). Shared pain: From empathy to synaesthesia. Neuroscience and Biobehavioral Reviews, 34(4), 500-512.

Fivush, R. (2011). The development of autobiographical memory. Annual Review of Psychology, 62, $559-582$.

Fivush, R., \& Nelson, K. (2004). Culture and language in the emergence of autobiographical memory. Psychological Science, 15(9), 573-577.

Fivush, R., \& Nelson, K. (2006). Parent-child reminiscing locates the self in the past. British Journal of Developmental Psychology, 24(1), 235-251.

Fox, J., Ralston, R. A., Cooper, C. K., \& Jones, K. A. (2015). Sexualized avatars lead to women's selfobjectification and acceptance of rape myths. Psychology of Women Quarterly, 39(3), 349-362.

Freeman, D., Evans, N., Lister, R., Antley, A., Dunn, G., \& Slater, M. (2014). Height, social comparison, and paranoia: An immersive virtual reality experimental study. Psychiatry Research, 218(3), $348-352$.

Fridland, E. (2011). The case for proprioception. Phenomenology and the Cognitive Sciences, 10(4), 521-540.

Frith, U., \& Frith, C. D. (2003). Development and neurophysiology of mentalizing. Philosophical Transactions of the Royal Society B: Biological Sciences, 358(1431), 459-473.

Galinsky, A. D., \& Ku, G. (2004). The effects of perspective-taking on prejudice: The moderating role of self-evaluation. Personality and Social Psychology Bulletin, 30, 594-604.

Galinsky, A. D., Ku, G., \& Wang, C. S. (2005). Perspective-taking and self-other overlap: Fostering social bonds and facilitating social coordination. Group Processes \& Intergroup Relations, 8(2), 109-124.

Gallagher, S. (2000). Philosophical conceptions of the self: Implications for cognitive science. Trends in Cognitive Sciences, 4(1), 14-21.

Gallese, V. (2007). Before and below "theory of mind": Embodied simulation and the neural correlates of social cognition. Philosophical Transactions of the Royal Society B: Biological Sciences, 362(1480), 659-669.

Gallese, V., Fadiga, L., Fogassi, L., \& Rizzolatti, G. (1996). Action recognition in the premotor cortex. Brain, 119(2), 593-609. 
Gawronski, B., \& Bodenhausen, G. V. (2006). Associative and propositional processes in evaluation: An integrative review of implicit and explicit attitude change. Psychological Bulletin, 132(5), 692-731.

Gibson, J. J. (1979). The ecological approach to visual perception. Boston: Houghton-Mifflin.

Goffman, E. (1959). The presentation of self in everyday life. Garden City, New Anchor: Doubleday Anchor Books.

Greenwald, A. G., \& Banaji, M. R. (1995). Implicit social cognition: Attitudes, self-esteem, and stereotypes. Psychological Review, 102(1), 4-27.

Groom, V., Bailenson, J. N., \& Nass, C. I. (2009). The influence of racial embodiment on racial bias in immersive virtual environments. Social Influence, 4(3), 231-248.

Gutierrez, B., Kaatz, A., Chu, S., Ramirez, D., Samson-Samuel, C., \& Carnes, M. (2014). "Fair Play": A videogame designed to address implicit race bias through active perspective taking. Games for Health Journal, 3(6), 371-378.

Gutsell, J. N., \& Inzlicht, M. (2010). Empathy constrained: Prejudice predicts reduced mental simulation of actions during observation of outgroups. Journal of Experimental Social Psychology, 46(5), $841-845$.

Gutsell, J. N., \& Inzlicht, M. (2012). Intergroup differences in the sharing of emotive states: Neural evidence of an empathy gap. Social Cognitive and Affective Neuroscience, 7(5), 596-603.

Gutsell, J. N., \& Inzlicht, M. (2013). Using EEG Mu-supression to explore group biases in motor resonance. In B. Derks, D. Scheepers, \& N. Ellemers (Eds.), Neuroscience of prejudice (pp. 278-298). London: Psychology Press.

Hakemulder, J. (2000). The moral laboratoryL Experiments examining the effects of reading literature on social perception and moral self-concept. Amsterdam: John Benjamins Publishing.

Hakemulder, J. (2008). Imagining what could happen: Effects of taking the role of a character on social cognition. In S. Zyngier, M. Bortolussi, A. Chesnokova, \& J. Auracher (Eds.), Directions in empirical literary studies: In honor of Willie van Peer (pp. 139-160). Amsterdam: John Benjamins Publishing.

Hale, J., \& de Hamilton, A. F. C. (2016). Cognitive mechanisms for responding to mimicry from others. Neuroscience and Biobehavioral Reviews, 63, 106-123.

Harris, L. T., \& Fiske, S. T. (2006). Dehumanizing the lowest of the low. Psychological Science, 17(10), 847-853.

Harris, L. T., \& Fiske, S. T. (2007). Social groups that elicit disgust are differentially processed in mPFC. Social Cognitive and Affective Neuroscience, 2(1), 45-51.

Hasler, B. S., Hirschberger, G., Shani-Sherman, T., \& Friedman, D. A. (2014). Virtual peacemakers: Mimicry increases empathy in simulated contact with virtual outgroup members. Cyberpsychology, Behavior and Social Networking, 17(12), 766-771.

Hasler, B. S., Spanlang, B., \& Slater, M. (2017). Virtual race transformation reverses racial ingroup bias. PLoS ONE, 12(4), e0174965.

Heatherton, T. F. (2006). Medial prefrontal activity differentiates self from close others. Social Cognitive and Affective Neuroscience, 1(1), 18-25.

Hove, M. J., \& Risen, J. L. (2009). It's all in the timing: Interpersonal synchrony increases affiliation. Social Cognition, 27(6), 949-960.

Hu, C., Di, X., Eickhoff, S. B., Zhang, M., Peng, K., Guo, H., et al. (2016). Distinct and common aspects of physical and psychological self-representation in the brain: A meta-analysis of self-bias in facial and self-referential judgements. Neuroscience and Biobehavioral Reviews, 61, 197-207.

Hunt, L. (2007). Inventing human rights: A history. New York, NY: W. W. Norton \& Co.

Hurley, S. L. (1998). Consciousness in action. London: Harvard University Press.

Husserl, E. (1983). Ideas pertaining to a pure phenomenology and to a phenomenological philosophy: First book: General introduction to a pure phenomenology. (F. Kersten, Trans.). Dordrecht: Kluwer.

Inzlicht, M., Gutsell, J. N., \& Legault, L. (2012). Mimicry reduces racial prejudice. Journal of Experimental Social Psychology, 48(1), 361-365.

Ionta, S., Heydrich, L., Lenggenhager, B., Mouthon, M., Fornari, E., Chapuis, D., et al. (2011). Multisensory mechanisms in temporo-parietal cortex support self-location and first-person perspective. Neuron, 70(2), 363-374.

Iruthayarajah, J., McIntyre, A., Cotoi, A., Macaluso, S., \& Teasell, R. (2017). The use of virtual reality for balance among individuals with chronic stroke: A systematic review and meta-analysis. Topics in Stroke Rehabilitation, 24(1), 68-79. 
Jabbi, M., Bastiaansen, J. A. C. J., \& Keysers, C. (2008). A common anterior insula representation of disgust observation, experience and imagination shows divergent functional connectivity pathways. PLOS ONE, 3(8), e2939.

James, W. (2014). The principles of psychology (Vol. 1). Global Grey.

Johnson, D. R., Jasper, D. M., Griffin, S., \& Huffman, B. L. (2013). Reading narrative fiction reduces Arab-Muslim prejudice and offers a safe haven from intergroup anxiety. Social Cognition, 31(5), $578-598$.

Jordan, C. H., Spencer, S. J., \& Zanna, M. P. (2005). Types of high self-esteem and prejudice: How implicit self-esteem relates to ethnic discrimination among high explicit self-esteem individuals. Personality and Social Psychology Bulletin, 31(5), 693-702.

Kawakami, K., Phills, C. E., Greenwald, A. G., Simard, D., Pontiero, J., Brnjas, A., et al. (2012). In perfect harmony: Synchronizing the self to activated social categories. Journal of Personality and Social Psychology, 102(3), 562-575.

Keysers, C., Kaas, J. H., \& Gazzola, V. (2010). Somatosensation in social perception. Nature Reviews Neuroscience, 11(6), 417-428.

Kilner, J. M., Friston, K. J., \& Frith, C. D. (2007). The mirror-neuron system: A Bayesian perspective. NeuroReport, 18(6), 619-623.

Kilner, J. M., Neal, A., Weiskopf, N., Friston, K. J., \& Frith, C. D. (2009). Evidence of mirror neurons in human inferior frontal gyrus. The Journal of Neuroscience, 29(32), 10153-10159.

Kilteni, K., Bergstrom, I., \& Slater, M. (2013). Drumming in immersive virtual reality: The body shapes the way we play. IEEE Transactions on Visualization and Computer Graphics, 19(4), 597-605.

Kim, K., \& Johnson, M. (2012). Extended self: Medial prefrontal activity during transient association of self and objects. Social Cognitive and Affective Neuroscience, 7(2), 199-207.

Knutson, K. M., Mah, L., Manly, C. F., \& Grafman, J. (2007). Neural correlates of automatic beliefs about gender and race. Human Brain Mapping, 28(10), 915-930.

Koopman, E. M., \& Hakemulder, F. (2015). Effects of literature on empathy and self-reflection: A theoretical-empirical framework. Journal of Literary Theory, 9(1), 79-111.

Kuhl, J., Quirin, M., \& Koole, S. L. (2015). Being someone: The integrated self as a neuropsychological system. Social and Personality Psychology Compass, 9(3), 115-132.

Kurzban, R., Tooby, J., \& Cosmides, L. (2001). Can race be erased? Coalitional computation and social categorization. Proceedings of the National Academy of Sciences, 98(26), 15387-15392.

Lai, C. K., Marini, M., Lehr, S. A., Cerruti, C., Shin, J.-E. L., Joy-Gaba, J. A., et al. (2014). Reducing implicit racial preferences: I. A comparative investigation of 17 interventions. Journal of Experimental Psychology: General, 143(4), 1765-1785.

Launay, J., Dean, R. T., \& Bailes, F. (2013). Synchronization can influence trust following virtual interaction. Experimental Psychology, 60(1), 53-63.

Lee, J.-E. R., Nass, C. I., \& Bailenson, J. N. (2013). Does the mask govern the mind?: Effects of arbitrary gender representation on quantitative task performance in avatar-represented virtual groups. Cyberpsychology, Behavior, and Social Networking, 53(9), 1689-1699.

Legrand, D. (2006). The bodily self: The sensori-motor roots of pre-reflective self-consciousness. Phenomenology and the Cognitive Sciences, 5(1), 89-118.

Leichtman, M. D., Wang, Q., \& Pillmer, D. B. (2003). Cultural variations in interdependence and autobiographical memory: Lessons from Korea, China, India, and the United States. In R. Fivush \& C. A. Haden (Eds.), Autobiographical memory and the construction of a narrative self: Developmental and cultural perspectives (pp. 73-98). Mahwah, NJ: Lawrence Erlbaum.

Lenggenhager, B., Tadi, T., Metzinger, T., \& Blanke, O. (2007). Video ergo sum: Manipulating bodily self-consciousness. Science, 317(5841), 1096-1099.

Leshikar, E. D., Cassidy, B. S., \& Gutchess, A. H. (2016). Similarity to the self influences cortical recruitment during impression formation. Cognitive, Affective, \& Behavioral Neuroscience, 16(2), 302-314.

Levitin, D. J. (2008). The world in six songs: How the musical brain created human nature. New York, NY: Penguin.

Lewis, M. L., Weber, R., \& Bowman, N. D. (2008). "They may be pixels, but they're MY pixels": Developing a metric of character attachment in role-playing video games. CyberPsychology \& Behavior, 11(4), 515-518.

Li, D. D., Liau, A. K., \& Khoo, A. (2013). Player-avatar identification in video gaming: Concept and measurement. Computers in Human Behavior, 29(1), 257-263. 
Liebert, R. M., Sobol, M. P., Copemann, C. D., Anderson, W., Friedman, J., Greenberg, C., et al. (1972). Effects of vicarious consequences and race of model upon imitative performance. Developmental Psychology, 6(3), 453-456.

Liew, S.-L., Han, S., \& Aziz-Zadeh, L. (2011). Familiarity modulates mirror neuron and mentalizing regions during intention understanding. Human Brain Mapping, 32(11), 1986-1997.

Limanowski, J., \& Blankenburg, F. (2013). Minimal self-models and the free energy principle. Frontiers in Human Neuroscience, 7, 547.

Limanowski, J., Lutti, A., \& Blankenburg, F. (2014). The extrastriate body area is involved in illusory limb ownership. NeuroImage, 86(1), 514-524.

Lindner, M., Rudorf, S., Birg, R., Falk, A., Weber, B., \& Fliessbach, K. (2013). Neural patterns underlying social comparisons of personal performance. Social Cognitive and Affective Neuroscience, 10(4), 569-576.

Lopez, C., Bieri, C. P., Preuss, N., \& Mast, F. W. (2012). Tactile and vestibular mechanisms underlying ownership for body parts: A non-visual variant of the rubber hand illusion. Neuroscience Letters, 511(2), 120-124.

Losin, E. A. R., Iacoboni, M., Martin, A., Cross, K. A., \& Dapretto, M. (2012). Race modulates neural activity during imitation. NeuroImage, 59(4), 3594-3603.

Ma, N., Baetens, K., Vandekerckhove, M., Van der Cruyssen, L., \& Van Overwalle, F. (2013). Dissociation of a trait and a valence representation in the mPFC. Social Cognitive and Affective Neuroscience, 9(10), 1506-1514.

Mackie, D. M., \& Smith, E. R. (2002). Intergroup emotions and the social self: Prejudice reconceptualized as differentiated reactions to out-groups. In J. P. Forgas \& K. D. Williams (Eds.), The social self: Cognitive, interpersonal and intergroup perspectives (pp. 309-326). New York, NY: Psychology Press.

Maister, L., Cardini, F., Zamariola, G., Serino, A., \& Tsakiris, M. (2015). Your place or mine: Shared sensory experiences elicit a remapping of peripersonal space. Neuropsychologia, 70, 455-461.

Maister, L., Sebanz, N., Knoblich, G., \& Tsakiris, M. (2013). Experiencing ownership over a darkskinned body reduces implicit racial bias. Cognition, 128(2), 170-178.

Maj, M., \& Kossowska, M. (2015). Prejudice toward outgroups as a strategy to deal with mortality threat: Simple reaction with a complex foundation. Theoria Et Historia Scientiarum, 12, 15-28.

Mar, R. A., \& Oatley, K. (2008). The function of fiction is the abstraction and simulation of social experience. Perspectives on Psychological Science, 3(3), 173-192.

Marini, M., Rubichi, S., \& Sartori, G. (2012). The role of self-involvement in shifting IAT effects. Experimental Psychology, 59(6), 348-354.

Markus, H. R., \& Kitayama, S. (2010). Cultures and selves: A cycle of mutual constitution. Perspectives on Psychological Science, 5(4), 420-430.

Markus, H. R., \& Wurf, E. (1987). The dynamic self-concept: A social psychological perspective. Annual Review of Psychology, 38(1), 299-337.

Mattan, B., Quinn, K. A., Apperly, I. A., Sui, J., \& Rotshtein, P. (2015). Is it always me first? Effects of self-tagging on third-person perspective-taking. Journal of Experimental Psychology. Learning, Memory, and Cognition, 41(4), 1100-1117.

McCambridge, J., de Bruin, M., \& Witton, J. (2012). The effects of demand characteristics on research participant behaviours in non-laboratory settings a systematic review. PLOS ONE, 7(6), e39116.

McCormick, C., St-Laurent, M., Ty, A., Valiante, T. A., \& McAndrews, M. P. (2015). Functional and effective hippocampal-neocortical connectivity during construction and elaboration of autobiographical memory retrieval. Cerebral Cortex, 25(5), 1297-1305.

McCoy, S. K., \& Major, B. (2003). Group identification moderates emotional responses to perceived prejudice. Personality and Social Psychology Bulletin, 29(8), 1005-1017.

McGlone, F., \& Reilly, D. (2010). The cutaneous sensory system. Neuroscience and Biobehavioral Reviews, 34(2), 148-159.

Mead, G. H. (1913). The social self. The Journal of Philosophy, Psychology and Scientific Methods, 10(14), 374-380.

Mead, G. H. (1935). Mind, self, and society from the standpoint of a social behaviorist. (C. W. Morris, Ed.). Chicago, Il: University of Chicago Press.

Merleau-Ponty, M. (2002). Phenomenology of perception. (C. Smith, Trans.). London: Routledge.

Mitchell, J. P., Macrae, C. N., \& Banaji, M. R. (2006). Dissociable medial prefrontal contributions to judgments of similar and dissimilar others. Neuron, 50(4), 655-663. 
Molenberghs, P., Cunnington, R., \& Mattingley, J. B. (2012). Brain regions with mirror properties: A meta-analysis of 125 human fMRI studies. Neuroscience and Biobehavioral Reviews, 36(1), 341-349.

Molnar-Szakacs, I., Wu, A. D., Robles, F. J., \& Iacoboni, M. (2007). Do you see what I mean? Corticospinal excitability during observation of culture-specific gestures. PLOS ONE, 2(7), e626.

Moore, W. E., Merchant, J. S., Kahn, L. E., \& Pfeifer, J. H. (2014). “Like me?”: Ventromedial prefrontal cortex is sensitive to both personal relevance and self-similarity during social comparisons. Social Cognitive and Affective Neuroscience, 9(4), 421-426.

Morita, T., Tanabe, H. C., Sasaki, A. T., Shimada, K., Kakigi, R., \& Sadato, N. (2014). The anterior insular and anterior cingulate cortices in emotional processing for self-face recognition. Social Cognitive and Affective Neuroscience, 9(5), 570-579.

Mukamel, R., Ekstrom, A. D., Kaplan, J. T., Iacoboni, M., \& Fried, I. (2010). Single-neuron responses in humans during execution and observation of actions. Current Biology, 20(8), 750-756.

Müller, B. C. N., Kühn, S., van Baaren, R. B., Dotsch, R., Brass, M., \& Dijksterhuis, A. (2011). Perspective taking eliminates differences in co-representation of out-group members' actions. Experimental Brain Research, 211(3-4), 423-428.

Murray, R. J., Debbané, M., Fox, P. T., Bzdok, D., \& Eickhoff, S. B. (2015). Functional connectivity mapping of regions associated with self- and other-processing. Human Brain Mapping, 36(4), 1304-1324.

Muscatell, K. A., Morelli, S. A., Falk, E. B., Way, B. M., Pfeifer, J. H., Galinsky, A. D., et al. (2012). Social status modulates neural activity in the mentalizing network. NeuroImage, 60(3), 1771-1777.

Neely, J. J., Heckel, R. V., \& Leichtman, H. M. (1973). The effect of race of model and response consequences to the model on imitiation in children. The Journal of Social Psychology, 89, $225-231$.

Neisser, U. (1991). Two perceptually given aspects of the self and their development. Developmental Review, 11, 197-209.

Nelson, K. (2003). Self and social functions: Individual autobiographical memory and collective narrative. Memory, 11(2), 125-136.

Ng, S. H., Han, S., Mao, L., \& Lai, J. C. L. (2010). Dynamic bicultural brains: fMRI study of their flexible neural representation of self and significant others in response to culture primes. Asian Journal of Social Psychology, 13(2), 83-91.

Nitschke, J. B., Sarinopoulos, I., MacKiewicz, K. L., Schaefer, H. S., \& Davidson, R. J. (2006). Functional neuroanatomy of aversion and its anticipation. NeuroImage, 29(1), 106-116.

Northoff, G., Heinzel, A., de Greck, M., Bermpohl, F., Dobrowolny, H., \& Panksepp, J. (2006). Selfreferential processing in our brain: A meta-analysis of imaging studies on the self. Neurolmage, 31(1), 440-457.

Northoff, G., Qin, P., \& Feinberg, T. E. (2011). Brain imaging of the self: Conceptual, anatomical and methodological issues. Consciousness and Cognition, 20(1), 52-63.

Nowak, K. L., Watt, J., \& Walther, J. B. (2005). The influence of synchrony and sensory modality on the person perception process in computer-mediated groups. Journal of Computer-Mediated Coтmunication, $10(3)$.

Oatley, K. (2016). Fiction: Simulation of social worlds. Trends in Cognitive Sciences, 20(8), 618-628.

Otten, S. (2003). "Me and us" or "us and them"? The self as a heuristic for defining minimal ingroups. European Review of Social Psychology, 13(1), 1-33.

Pacherie, E. (2014). How does it feel to act together? Phenomenology and the Cognitive Sciences, 13(1), 25-46.

Paluck, E. L. (2007). Reducing intergroup prejudice and conflict with the mass media: A field experiment in Rwanda. In Dissertation Abstracts International: Section B: The Sciences and Engineering, 68(6B), p. 4179.

Payne, S., Tsakiris, M., \& Maister, L. (2017). Can the self become another? Investigating the effects of self-association with a new facial identity. The Quarterly Journal of Experimental Psychology, 70(6), 1085-1097.

Peck, T. C., Seinfeld, S., Aglioti, S. M., \& Slater, M. (2013). Putting yourself in the skin of a black avatar reduces implicit racial bias. Consciousness and Cognition, 22(3), 779-787.

Peña, J., Hancock, J. T., \& Merola, N. A. (2009). The priming effects of avatars in virtual settings. Communication Research, 36(6), 838-856. 
Peña, J., Khan, S., \& Alexopoulos, C. (2016). I am what I see: How avatar and opponent agent body size affects physical activity among men playing exergames. Journal of Computer-Mediated Coтmunication, 21, 195-209.

Perugini, M., \& Leone, L. (2009). Implicit self-concept and moral action. Journal of Research in Personality, 43(5), 747-754.

Petkova, V. I., Björnsdotter, M., Gentile, G., Jonsson, T., Li, T.-Q., \& Ehrsson, H. H. (2011). From partto whole-body ownership in the multisensory brain. Current Biology, 21(13), 1118-1122.

Petkova, V. I., \& Ehrsson, H. H. (2008). If I were you: Perceptual illusion of body swapping. PLoS ONE, $3(12), \mathrm{e} 3832$.

Pfeiffer, C., Lopez, C., Schmutz, V., Duenas, J. A., Martuzzi, R., \& Blanke, O. (2013). Multisensory origin of the subjective first-person perspective: Visual, tactile, and vestibular mechanisms. PLoS ONE, 8(4), e61751.

Pillmer, D. B. (1998). Momentous events, vivid memories: How unforgettable moments help us understand the meaning of our lives. Cambridge, MA: Harvard University Press.

Pinker, S. (2011). The better angels of our nature: The decline of violence in history and its causes. London: Allen Lane.

Polkinghorne, D. E. (1991). Narrative and self-concept. Journal of Narrative and Life History, 1, 135-153.

Powell, L. J., Macrae, C. N., Cloutier, J., Metcalfe, J., \& Mitchell, J. P. (2010). Dissociable neural substrates for agentic versus conceptual representations of self. Journal of Cognitive Neuroscience, 22(10), 2186-2197.

Powers, K. E., Wagner, D. D., Norris, C. J., \& Heatherton, T. F. (2013). Socially excluded individuals fail to recruit medial prefrontal cortex for negative social scenes. Social Cognitive and Affective Neuroscience, 8(2), 151-157.

Prebble, S. C., Addis, D. R., \& Tippett, L. J. (2013). Autobiographical memory and sense of self. Psychological Bulletin, 139(4), 815-840.

Prestwich, A., Perugini, M., Hurling, R., \& Richetin, J. (2010). Using the self to change implicit attitudes. European Journal of Social Psychology, 71, 61-71.

Rauchbauer, B., Majdandžić, J., Stieger, S., \& Lamm, C. (2016). The modulation of mimicry by ethnic group-membership and emotional expressions. PLOS ONE, 11(8), e0161064.

Reddish, P., Bulbulia, J., \& Fischer, R. (2014). Does synchrony promote generalized prosociality? Religion, Brain and Behavior, 4(1), 3-19.

Richeson, J. A., Baird, A. A., Gordon, H. L., Heatherton, T. F., Wyland, C. L., Trawalter, S., et al. (2003). An fMRI investigation of the impact of interracial contact on executive function. Nature Neuroscience, 6(12), 1323-1328.

Riemer, M., Kleinböhl, D., Hölzl, R., \& Trojan, J. (2013). Action and perception in the rubber hand illusion. Experimental Brain Research, 229(3), 383-393.

Riva, G., Banos, R. M., Botella, C., Mantovani, F., \& Gaggioli, A. (2016). Transforming experience: The potential of augmented reality and virtual reality for enhancing personal and clinical change. Frontiers in Psychiatry, 7, 164.

Ronquillo, J., Denson, T. F., Lickel, B., Lu, Z.-L., Nandy, A., \& Maddox, K. B. (2007). The effects of skin tone on race-related amygdala activity: An fMRI investigation. Social Cognitive and Affective Neuroscience, 2(1), 39-44.

Rosenberg, R. S., Baughman, S. L., \& Bailenson, J. N. (2013). Virtual superheroes: Using superpowers in virtual reality to encourage prosocial behavior. PLOS ONE, 8(1), e55003.

Rudman, L. A., Dohn, M. C., \& Fairchild, K. (2007). Implicit self-esteem compensation: Automatic threat defense. Journal of Personality and Social Psychology, 93(5), 798-813.

Sacheli, L. M., Christensen, A., Giese, M. A., Taubert, N., Pavone, E. F., Aglioti, S. M., et al. (2015). Prejudiced interactions: Implicit racial bias reduces predictive simulation during joint action with an out-group avatar. Scientific Reports, 5, 8507.

Sanfey, A. G., Rilling, J. K., Aronson, J. A., Nystrom, L. E., \& Cohen, J. D. (2003). The neural basis of economic decision-making in the Ultimatum Game. Science, 300(5626), 1755-1758.

Santiesteban, I., Banissy, M. J., Catmur, C., \& Bird, G. (2012). Enhancing social ability by stimulating right temporoparietal junction. Current Biology, 22(23), 2274-2277.

Sartre, J.-P. (2003). Being and nothingness. (H. E. Barnes, Trans.). Oxford: Routledge.

Schaefer, M., Heinze, H.-J., \& Rotte, M. (2012). Embodied empathy for tactile events: Interindividual differences and vicarious somatosensory responses during touch observation. NeuroImage, 60(2), 952-957. 
Schilbach, L. (2015). The neural correlates of social cognition and social interaction. Brain Mapping, 3, 159-164.

Sellaro, R., Derks, B., Nitsche, M. A., Hommel, B., Van Den Wildenberg, W. P. M., Van Dam, K., et al. (2015). Reducing prejudice through brain stimulation. Brain Stimulation, 8(5), 891-897.

Serino, A., Giovagnoli, G., \& Làdavas, E. (2009). I feel what you feel if you are similar to me. PLoS ONE, 4(3), e4930.

Seth, A. K., Suzuki, K., \& Critchley, H. D. (2011). An interoceptive predictive coding model of conscious presence. Frontiers in Psychology, 2, 395.

Sforza, A., Bufalari, I., Haggard, P., \& Aglioti, S. M. (2010). My face in yours: Visuo-tactile facial stimulation influences sense of identity. Social Neuroscience, 5(2), 148-162.

Sheng, F., \& Han, S. (2012). Manipulations of cognitive strategies and intergroup relationships reduce the racial bias in empathic neural responses. NeuroImage, 61(4), 786-797.

Shih, M., Wang, E., Trahan Bucher, A., \& Stotzer, R. (2009). Perspective taking: Reducing prejudice towards general outgroups and specific individuals. Group Processes \& Intergroup Relations, 12(5), $565-577$.

Simmons, W. K., Avery, J. A., Barcalow, J. C., Bodurka, J., Drevets, W. C., \& Bellgowan, P. (2013). Keeping the body in mind: Insula functional organization and functional connectivity integrate interoceptive, exteroceptive, and emotional awareness. Human Brain Mapping, 34(11), 2944-2958.

Slater, M., Perez-Marcos, D., Ehrsson, H. H., \& Sanchez-Vives, M. V. (2009). Inducing illusory ownership of a virtual body. Frontiers in Neuroscience, 3(2), 214-220.

Souppouris, A. (2014). Virtual reality made me believe I was someone else. Retrieved October 10, 2016, from http://www.theverge.com/2014/3/24/5526694/virtual-reality-made-me-believe-i-was-someone-else

Spencer, S. J., Fein, S., Wolfe, C., Fong, C., \& Duinn, M. (1998). Automatic activation of stereotypes: The role of self-image threat. Personality and Social Psychology Bulletin, 24(11), 1139-1152.

Spreng, R. N., Mar, R. A., \& Kim, A. S. N. (2009). The common neural basis of autobiographical memory, prospection, navigation, theory of mind, and the default mode: A quantitative metaanalysis. Journal of Cognitive Neuroscience, 21(3), 489-510.

Strawson, G. (1999). The self and the SESMET. Journal of Consciousness Studies, 6(4), 99-135.

Sugiura, M. (2013). Associative account of self-cognition: Extended forward model and multi-layer structure. Frontiers in Human Neuroscience, 7, 535.

Sui, J., He, X., \& Humphreys, G. W. (2012). Perceptual effects of social salience: Evidence from selfprioritization effects on perceptual matching. Journal of Experimental Psychology: Human Perception and Performance, 38(5), 1105-1117.

Sui, J., \& Humphreys, G. W. (2015). The integrative self: How self-reference integrates perception and memory. Trends in Cognitive Sciences, 19(12), 719-728.

Sui, J., Rotshtein, P., \& Humphreys, G. W. (2013). Coupling social attention to the self forms a network for personal significance. Proceedings of the National Academy of Sciences, 110(19), 7607-7612.

Svoboda, E., McKinnon, M. C., \& Levine, B. (2006). The functional neuroanatomy of autobiographical memory: A meta-analysis. Neuropsychologia, 44(12), 2189-2208.

Tajfel, H. (1974). Social identity and intergroup behaviour. Social Science Information, 13(2), 65-93.

Tajfel, H. (1982). Social psychology of intergroup relations. Annual Review of Psychology, 26, 1-39.

Teo, W. P., Muthalib, M., Yamin, S., Hendy, A. M., Bramstedt, K., Kotsopoulos, E., et al. (2016). Does a combination of virtual reality, neuromodulation and neuroimaging provide a comprehensive platform for neurorehabilitation? A narrative review of the literature. Frontiers in Human Neuroscience, 10, 284.

Todd, A. R., \& Burgmer, P. (2013). Perspective taking and automatic intergroup evaluation change: Testing an associative self-anchoring account. Journal of Personality and Social Psychology, 104(5), 786-802.

Tsakiris, M. (2008). Looking for myself: Current multisensory input alters self-face recognition. PLoS ONE, 3(12), e4040.

Tsakiris, M., Costantini, M., \& Haggard, P. (2008). The role of the right temporo-parietal junction in maintaining a coherent sense of one's body. Neuropsychologia, 46(12), 3014-3018.

Tsakiris, M., Hesse, M. D., Boy, C., Haggard, P., \& Fink, G. R. (2007). Neural signatures of body ownership: A sensory network for bodily self-consciousness. Cerebral Cortex, 17(10), 2235-2244.

Tunçgenç, B., \& Cohen, E. (2016). Movement synchrony forges social bonds across group divides. Frontiers in Psychology, 7, 782.

Valdesolo, P., \& Desteno, D. (2011). Synchrony and the social tuning of compassion. Emotion, 11(2), 262-266. 
Vallar, G., \& Ronchi, R. (2009). Somatoparaphrenia: A body delusion: A review of the neuropsychological literature. Experimental Brain Research, 192(3), 533-551.

Valmaggia, L. R., Latif, L., Kempton, M. J., \& Rus-Calafell, M. (2016). Virtual reality in the psychological treatment for mental health problems: An systematic review of recent evidence. Psychiatry Research, 236, 189-195.

Van Bavel, J. J., \& Cunningham, W. A. (2009). Self-categorization with a novel mixed-race group moderates automatic social and racial biases. Personality and Social Psychology Bulletin, 35(3), 321-335.

van der Schalk, J., Fischer, A. H., Doosje, B., Wigboldus, D., Hawk, S., Rotteveel, M., et al. (2011). Convergent and divergent responses to emotional displays of ingroup and outgroup. Emotion, 11(2), 286-298.

Vescio, T. K., Sechrist, G. B., \& Paolucci, M. P. (2003). Perspective taking and prejudice reduction: The mediational role of empathy arousal and situational attributions. European Journal of Social Psychology, 33(4), 455-472.

Vezzali, L., Stathi, S., Giovannini, D., Capozza, D., \& Trifiletti, E. (2015). The greatest magic of Harry Potter: Reducing prejudice. Journal of Applied Social Psychology, 45(2), 105-121.

Walsh, L. D., Moseley, G. L., Taylor, J. L., \& Gandevia, S. C. (2011). Proprioceptive signals contribute to the sense of body ownership. Journal of Physiology, 589(12), 3009-3021.

Wang, C., Wu, B., Liu, Y., Wu, X., \& Han, S. (2014). Challenging emotional prejudice by changing selfconcept: Priming independent self-construal reduces racial in-group bias in neural responses to other's pain. Social Cognitive and Affective Neuroscience, 10(9), 1195-1201.

Weisbuch, M., \& Ambady, N. (2008). Affective divergence: Automatic responses to others' emotions depend on group membership. Journal of Personality and Social Psychology, 95(5), 1063-1079.

Wheeler, S. C., Demarree, K. G., \& Petty, R. E. (2007). Understanding the role of the self in prime-tobehavior effects: The active-self account. Personality and Social Psychology Review, 11(3), 234-261.

White, R. C., Davies, A. M. A., \& Davies, M. (2011). Two hands are better than one: A new assessment method and a new interpretation of the non-visual illusion of self-touch. Consciousness and Cognition, 20(3), 956-964.

Wiltermuth, S. S., \& Heath, C. (2009). Synchrony and cooperation. Psychological Science, 20(1), 1-5.

Xiang, T., Lohrenz, T., \& Montague, P. R. (2013). Computational substrates of norms and their violations during social exchange. The Journal of Neuroscience, 33(3), 1099-1108.

Xu, X., Zuo, X., Wang, X., \& Han, S. (2009). Do you feel my pain? Racial group membership modulates empathic neural responses. The Journal of Neuroscience, 29(26), 8525-8529.

Yabar, Y., Johnston, L., Miles, L. K., \& Peace, V. (2006). Implicit behavioral mimicry: Investigating the impact of group membership. Journal of Nonverbal Behavior, 30(3), 97-113.

Yee, N., \& Bailenson, J. N. (2006). Walk a mile in digital shoes: The impact of embodied perspectivetaking on the reduction of negative stereotyping in immersive virtual environments. Proceedings of PRESENCE, 246, 147-156.

Yee, N., \& Bailenson, J. N. (2007). The proteus effect: The effect of transformed self representation on behavior. Human Communication Research, 33(3), 271-290.

Yee, N., Bailenson, J. N., \& Ducheneaut, N. (2009). The proteus effect: Implications of transformed digital self-representation on online and offline behavior. Communication Research, 36(2), 285-312.

Zahavi, D. (2005). Subjectivity and selfhood: Investigating the first-person perspective. Cambridge, MA: MIT Press.

Zheng, Z. Z., Macdonald, E. N., Munhall, K. G., \& Johnsrude, I. S. (2011). Perceiving a stranger's voice as being one's own: A "rubber voice" illusion? PLOS ONE, 6(4), e18655.

Zhu, Y., Zhang, L., Fan, J., \& Han, S. (2007). Neural basis of cultural influence on self-representation. NeuroImage, 34(3), 1310-1316. 